\title{
Therapeutic Effect and Mechanism Study of Rhodiola wallichiana var. cholaensis Injection to Acute Blood Stasis Using Metabolomics Based on UPLC-Q/TOF-MS
}

\author{
Nan Ran $\left(D,{ }^{1}\right.$ Zhiqiang Pang, ${ }^{1}$ Xuewa Guan, ${ }^{1}$ Guoqiang Wang, ${ }^{1}$ Jinping Liu, ${ }^{2}$ Pingya Li, ${ }^{2}$ \\ Jingtong Zheng $\left(\mathbb{D},{ }^{1}\right.$ and Fang Wang $\mathbb{D}^{1}$ \\ ${ }^{1}$ Department of Pathogen Biology, College of Basic Medical Sciences, Jilin University, Changchun 130021, China \\ ${ }^{2}$ Research Center of Natural Drug, School of Pharmaceutical Sciences, Jilin University, Changchun 130021, China \\ Correspondence should be addressed to Fang Wang; wf@jlu.edu.cn
}

Received 4 May 2019; Revised 15 August 2019; Accepted 8 October 2019; Published 3 November 2019

Academic Editor: Sebastian Granica

Copyright (C) 2019 Nan Ran et al. This is an open access article distributed under the Creative Commons Attribution License, which permits unrestricted use, distribution, and reproduction in any medium, provided the original work is properly cited.

\begin{abstract}
In traditional Chinese medicine theory, blood stasis syndrome (BSS), characterized by blood flow retardation and blood stagnation, is one of the main pathologic mechanisms and clinical syndromes of cardiovascular diseases (CVDs). Rhodiola wallichiana var. cholaensis injection (RWCI) is made from dry roots and stems of RWC via the processes of decoction, alcohol precipitation, filtration, and dilution. Studies indicated the extracts of RWC could alleviate CVDs; however, the mechanism had not been illustrated. In the present study, the acute blood stasis rat model was established to investigate the pathogenesis of BSS and the therapeutic mechanism of RWCI against BSS. Hemorheological parameters (whole blood viscosity and plasma viscosity) and inflammatory factors (TNF- $\alpha$ and IL-6) were used to evaluate the success of the BSS rat model and RWCI efficacy. 14 and 33 differential metabolites were identified from plasma and urine samples using the metabolomics approach based on ultrahighperformance liquid chromatography coupled with quadrupole time-of-flight mass spectrometry. The results of multivariate analysis displayed that there were significant separations among model, control, and treatment groups, but the high-dose RWCI treatment group was closer to the control group. 9 perturbed metabolic pathways were related to BSS's development and RWCI intervention. 5 metabolic pathways (arachidonic acid metabolism, linoleic acid metabolism, alpha-linolenic acid metabolism, retinol metabolism, and steroid hormone biosynthesis) showed apparent correlations. These differential metabolites and perturbed metabolic pathways might provide a novel view to understand the pathogenesis of BSS and the pharmacological mechanism of RWCI.
\end{abstract}

\section{Introduction}

In recent years, cardiovascular diseases (CVDs) have become a leading cause of death worldwide [1]. In order to control the increasing morbidity and mortality of CVDs, it is necessary to develop novel and effective therapeutic strategies. Integrating the multichannel, multicomponent, and multitarget characteristics, traditional Chinese medicine (TCM) based on cardiovascular drugs has been widely used to prevent and treat CVDs $[2,3]$. In TCM theory, blood stasis syndrome (BSS) is one of the main pathologic mechanisms and clinical syndromes of CVDs [4]. BSS is featured by blood flow retardation and blood stagnation and caused by several factors, for example, Qi deficiency, Qi stagnation, phlegm turbidity, cold coagulation, and blood insufficiency $[5,6]$. As one of the common TCM symptoms, BSS is always accompanied by the change of clinical manifestations and signs, hemorheological disorder, and vascular endothelial cell (VEC) damage and inflammation [7, 8]. Furthermore, BSS may result in multiple organ dysfunction [9]. Previous studies have reported that TCM might significantly improve the BSS by activating circulation to remove blood stasis [10]. However, the pathophysiological mechanisms have not been elucidated. 
Rhodiola, growing in cool and moist areas, has various species including Rhodiola wallichiana var. cholaensis (RWC), Rhodiola rosea, Rhodiola crenulata, and Rhodiola sachalinensis [11]. Currently, Rhodiola has been applied to improve coronary heart disease, bronchial asthma, and chronic fatigue syndrome in clinical practice [12]. RWC has broad therapeutic effects on human diseases, such as neuroprotective, antidepressive, antitumour, and cardioprotective activities, with milder effects and less frequent adverse effects [13]. The chemical constituents of RWC injection (RWCI) have been comprehensively studied. On the one hand, a total of 49 compounds including 10 organic acids, 9 phenylethanoids, 10 phenylpropanoids, 2 flavonoid glycosides, 7 monoterpene glycosides, 7 octylglycosides, and 4 other types of compounds were identified using the ultrahigh-performance liquid chromatography coupled with quadrupole time-of-flight mass spectrometry (UPLC-Q/ TOF-MS) analysis method [14]. On the other hand, the fingerprint RWCI had been established by HPLC [15]. Because of the diversity of components and biological activities, RWC has various functions in medicine and health, for instance, immune modulation, enhanced immunity [16], antitumor activity [17], and strengthening the ability of antianoxia [18]. RWCI is made from dry roots and stems of RWC via the processes of decoction, alcohol precipitation, filtration, and dilution. Recently, a study revealed that the RWC capsule had a significant effect on angina pectoris, one type of coronary heart disease [19]. RWCI is used to promote blood circulation and clear blood stasis, while the precise pharmacological mechanisms of the prevention and treatment of BSS are rarely reported.

Metabolomics, an emerging omics research approach, is employed to analyse the change of low-molecular-weight metabolites $(<1 \mathrm{kDa})$ in the complex biosystems influenced by endogenous and exogenous factors [20]. As a branch of system biology, metabolomics adopts a holistic research strategy to illustrate the metabolic process of the organism [21]. Unlike the genome and proteome, metabolome, a bridge between the genome and the phenotype, is viewed as the endpoint of biological processes [22]. The comprehensive thinking of metabolomics is consistent with the viewpoint of "whole," "dynamic process," and "dialectics" in TCM theory. Therefore, metabolomics is widely used to investigate the pharmacological effect of TCM, identify the compounds of Chinese herbs, and discover potential biomarkers related to diseases [23]. Especially, the UPLC-Q/TOF-MS method was developed as a very effective biochemical analytical tool for precise identification of important biomarkers, especially for largescale untargeted metabolic profiling in complex biological samples because of the high resolution of chromatographic peaks, high throughput, and high sensitivity for complex mixtures [24-26]. In conclusion, metabolomics is a beneficial and worthwhile tool to explore the potential therapeutic mechanism of TCM and the pathogenesis of diseases.

To further explore the pathogenesis of BSS and the therapeutic mechanisms of RWCI against BSS, in the present study, the acute blood stasis rat model was established by icewater bath combined with adrenaline. The success of the model and RWCI therapeutic effect were evaluated by hemorheological parameters (whole blood viscosity and plasma viscosity) and inflammatory factors. Subsequently, we analysed the change of metabolic profiling in plasma and urine samples from experimental rats using metabolomics based on UPLC-Q/TOF-MS. Potential distinct metabolites were used for further metabolic pathway analysis. Differential metabolites and perturbed metabolic pathways have been hypothesized to illustrate the pathogenesis of BSS and the molecular mechanism of RWCI against BSS.

\section{Materials and Methods}

2.1. Experimental Reagents. RWCI (Lot No. X1001170405) was provided by Tonghua Yusheng Pharmaceutical Co., Ltd. (Jilin, China; Z20060361). Adrenaline hydrochloride injections (Lot No. 1611281) were manufactured by Suicheng Pharmaceutical Co., Ltd. (Henan, China; H41021054). Compound danshen tablets (CDSTs) (Lot No. C17A050) were obtained from Hutchison Whampoa Guangzhou Baiyun Shan Chinese Medicine Co., Ltd. (Guangzhou, China; Z44023372). HPLC-grade acetonitrile (Lot No. 175165) was purchased from Fisher Chemical Company (Shanghai, China). Heparin sodium (Lot No. 1304049) and pentobarbital sodium (Lot No. 3289010) were produced by Sigma-Aldrich (St. Louis, MO, USA). Purified deionized water from the Milli-Q water purification system (Millipore, Billerica, MA, USA) was used for the preparation of aqueous solutions. All-trans-retinoic acid (Lot No. 57F-3895), 9-cisretinoic acid (Lot No. 107F-1633), 12(s)-HPETE (Lot No. 58F-1750), and 5-HPETE (Lot No. 241F-1496) were purchased from Sigma-Aldrich (St. Louis, MO, USA). The ELISA kits of tumor necrosis factor alpha (TNF- $\alpha$ ) (Lot No. A28280745) and interleukin-6 (IL-6) (Lot No. A20680741) were purchased from Multisciences (Lianke) Biotech, CO., Ltd. (Guangzhou, China).

2.2. Experimental Rats and Animal Model. Forty-eight healthy female SD rats $(200 \pm 20 \mathrm{~g})$ were purchased from YiSi Animal Co., Ltd. (Jilin, China; Certificate No. SCXK (JI)2016-0003). Those animals were raised in a comfortable environment with a temperature and humidity of $25 \pm 2{ }^{\circ} \mathrm{C}$ and $50 \pm 10 \%$. Moreover, 12-hour circulation of light and dark was supplied. Enough chow and water were provided for rats during the experiment. The whole experiment followed Nutrition Guidelines for the Care and Use of Laboratory Animals and was approved by the Institutional Animal Care and Use Committee of Jilin University (No. SCXK-2013-0001, September 24, 2017).

The animals were randomly and equally divided into six groups including the model group $(\mathrm{M})$, control group $(\mathrm{C})$, high-dose $(5 \mathrm{ml} / \mathrm{kg} /$ day, $\mathrm{TH})$, middle-dose $(2.5 \mathrm{ml} / \mathrm{kg} /$ day, TM), and low-dose $(1.25 \mathrm{ml} / \mathrm{kg} /$ day, TL) RWCI treatment groups, and CDST group $(0.3 \mathrm{~g} / \mathrm{kg} /$ day) (positive control group) after adapting to the environment for seven days. The corresponding administration dose was calculated by referring to Pharmacopoeia of the People's Republic of China (2015 edition, Volume I) [27] and Experimental Methodology of Pharmacology compiled by Wei et al. [28]. The BSS 
rat model was established according to previous researches $[29,30]$. In brief, the treated animals were administered by gavage with corresponding doses of RWCI and CDSTs for eight days, respectively. The animals in model and control groups were given normal saline. Except those in the control group, rats in other groups were placed in ice-cold water for 5 min daily for eight consecutive days and made to keep their heads above the water surface. At the eighth day, rats, except those in the control group, were subcutaneously injected adrenaline twice at a dose of $0.8 \mathrm{mg} / \mathrm{kg}$ in four-hour intervals. Moreover, ice-cold bath was performed after two hours of the first injection. Rats in the control group received an equal volume of saline by the way of subcutaneous injection.

2.3. Sample Collection and Processing. After $30 \mathrm{~min}$ of the last injection of epinephrine, the rats in treatment groups were administered by gavage with corresponding doses of RWCI and CDSTs. Urine samples from every rat were collected for twelve hours by putting rats in metabolic cages and were then immediately stored at $-80^{\circ} \mathrm{C}$ until analysis. In this period, urine samples were stored at $4^{\circ} \mathrm{C}$. Then, all rats were intraperitoneally anesthetized with $3 \%$ pentobarbital sodium $(1.5 \mathrm{~mL} / \mathrm{kg})$ after the collection of urine samples. Blood samples, from the abdominal aorta, were collected and mixed with $1 \%$ heparin sodium dilution $(10 \mathrm{mg} / \mathrm{mL})$ in $5 \mathrm{~mL}$ vacuum blood-collection tubes with heparin sodium anticoagulation for the hemorheological test and metabolomics analysis, respectively, after the collection of urine samples. Plasma was separated from blood by centrifuging at $3000 \mathrm{rpm}$ for $10 \mathrm{~min}$ at $4^{\circ} \mathrm{C} .1 \mathrm{~mL}$ was used for the plasma viscosity test. $200 \mu \mathrm{L}$ was immediately stored at $-80^{\circ} \mathrm{C}$ for metabolomics analysis.

2.4. Hemorheology and Inflammatory Factor Detection. Whole blood viscosity (at high, middle, and low shear rates of $120 \mathrm{~s}^{-1}, 60 \mathrm{~s}^{-1}$, and $10 \mathrm{~s}^{-1}$ ) and plasma viscosity (at the shear rate of $120 \mathrm{~s}^{-1}$ ) were detected by using an LBY-N6 blood viscometer (Precil, Beijing, China) at $37^{\circ} \mathrm{C}$. These experiments were completed within 2 hours after blood sample collection. The levels of cytokines (TNF- $\alpha$ and IL-6) in plasma samples were detected using the corresponding ELISA kits as recommended by the product's instructions.

2.5. Sample Preparation for Metabolomics Analysis. Both plasma and urine samples were thawed on ice before metabolomics analysis. Plasma samples were centrifuged at $3,000 \mathrm{rpm}$ for $10 \mathrm{~min}$ at $4^{\circ} \mathrm{C}$. The mixture of plasma $(200 \mu \mathrm{L})$ and acetonitrile $(600 \mu \mathrm{L})$ was vortexed for $3 \mathrm{~min}$. Next, they were settled at room temperature for $10 \mathrm{~min}$ and then centrifuged at $12,000 \mathrm{rpm}$ for $10 \mathrm{~min}$ at $4^{\circ} \mathrm{C}$. After the precooling at $-80^{\circ} \mathrm{C}, 500 \mu \mathrm{L}$ supernatant was lyophilized at $-60^{\circ} \mathrm{C}$ and $10.0 \mathrm{pa}$ air pressure for 18 hours. The residue was redissolved in $100 \mu \mathrm{L} 80 \%$ acetonitrile and then centrifuged at $12,000 \mathrm{~g}$ for $15 \mathrm{~min}$ at $4^{\circ} \mathrm{C}$. An aliquot of $3 \mu \mathrm{L}$ was injected for metabolomics analysis. Urine samples were centrifuged at $12,000 \mathrm{rpm}$ for $10 \mathrm{~min}$ at $4^{\circ} \mathrm{C}$. Moreover, the supernatant $(1 \mathrm{~mL})$ was separated for metabolomics analysis.

2.6. UPLC-Q/TOF-MS Conditions. The UPLC-Q/TOF-MSbased metabolomics analysis of plasma and urine samples was conducted by the Waters ACQUITY UPLC system (Waters Corporation, Milford, MA, USA) equipped with the BEH C18 column $(2.1 \mathrm{~mm} \times 100 \mathrm{~mm}, 1.7 \mathrm{~mm}$; Waters Corporation, Milford, MA, USA), coupled with the Waters Xevo G2-S Q/TOF mass spectrometer (Waters Corporation, Milford, MA, USA) with an electrospray ionization in both positive $\left(\mathrm{ESI}^{+}\right)$and negative $\left(\mathrm{ESI}^{-}\right)$ion modes. Column temperature was set as $30^{\circ} \mathrm{C}$, sample manager temperature as $15^{\circ} \mathrm{C}$, sample volume as $5 \mu \mathrm{L}$, and flow rate of mobile phases in the UPLC system as $0.4 \mathrm{~mL} / \mathrm{min}$. The mobile phases consisted of eluent A ( $0.1 \%$ formic acid in water, $\mathrm{v} / \mathrm{v})$ and eluent B $(0.1 \%$ formic acid in acetonitrile, $v / v)$. The gradient procedure was optimized as follows: $10 \% \mathrm{~B}$ from 0 to $2 \mathrm{~min}$, $10-90 \%$ B from 2 to $26 \mathrm{~min}, 90 \%$ B from 26 to $28 \mathrm{~min}$, $90-10 \%$ B from 28 to $28.1 \mathrm{~min}$, and $10 \%$ B from 28.1 to $30 \mathrm{~min}$. The parameters of chromatography and mass spectrometry were based on the previous study of our research team [31]. In brief, the capillary voltages and sample cone voltage were $2.6 \mathrm{kV}$ and $40 \mathrm{~V}$ in the $\mathrm{ESI}^{+}$mode, respectively. However, they were $2.2 \mathrm{kV}$ and $40 \mathrm{~V}$ in the $\mathrm{ESI}^{-}$ mode. The cone gas flow rate was $50 \mathrm{~L} / \mathrm{h}$ under the $120^{\circ} \mathrm{C}$ source temperature condition. The desolvation gas rate flow was $800 \mathrm{~L} / \mathrm{h}$ at $300^{\circ} \mathrm{C}$ desolvation temperature. Low and high collision energies were set as $6.0 \mathrm{~V}$ and $20-40 \mathrm{~V}$, respectively, in the MSE mode of mass spectrometry. Leucine-enkephalin (concentration: $300 \mathrm{ng} / \mathrm{mL}$; flow rate: $20 \mu \mathrm{L} / \mathrm{min}$ ) was regarded as the lock-mass solution.

Quality control (QC) samples of plasma and urine were prepared by mixing the equal volume $(20 \mu \mathrm{L})$ of all preprocessed plasma and urine samples, respectively, which were used to ensure the stability and repeatability of UPLCQ/TOF-MS analysis. QC samples were continuously tested six times in $\mathrm{ESI}^{+}$and $\mathrm{ESI}^{-}$modes, respectively, before the test of plasma and urine samples, which were used to evaluate the system method repeatability. The QCs were closely clustered in both positive and negative ion modes in both urinary and plasma samples, indicating the system method was stable. The intermediate precision and repeatability of the systematic method were validated by selecting ten chromatographic peaks of ions with high abundances in $\mathrm{ESI}^{+}$and $\mathrm{ESI}^{-}$modes, respectively, from the QC sequencing datasheet, covering the whole analysis process. Then, QC samples were tested again prior to the test of every group in plasma and urine samples to ensure the stability and suitability of MS analysis.

2.7. Data Processing. The raw data from metabolomics analysis were processed by the MarkerLynx XS v4.1 software (Waters, Milford, CT, USA) for alignment, deconvolution, data reduction, normalization, etc., which exported datasheet about the pair of mass and retention time with the corresponding intensities of all detected peaks. The main parameters were based on previous research. Retention time 
was set as 0 to $30 \mathrm{~min}$, mass as $100 \mathrm{Da}$ to $1,200 \mathrm{Da}$, mass tolerance as 0.10 , minimum intensity as $5 \%$, marker intensity threshold as 2,000 , mass window as $0.10 \mathrm{Da}$, retention time window as $0.20 \mathrm{~min}$, and noise elimination level as 6 [31].

The data in the datasheet were performed for multivariate analysis (principle component analysis (PCA) and orthogonal projections to latent structures discriminant analysis (OPLS-DA)) using the MarkerLynx XS v4.1 software and SIMCA-P software (v14.1; Umetrics, Umeå, Sweden). OPLS-DA, producing S-plots and permutation plots, contributed to finding significantly distinct metabolites in the metabolic process. The quality parameters (R2X, R2Y, and Q2) were employed to access the ability of the main components of variables to build models and samples, fitness between the model and samples, and prediction ability of the systematic model. Variable importance of project (VIP) values were also estimated statistically to indicate a significant difference between the groups. Metabolites were chosen based on VIP values (VIP $>1$ ) from the OPLS-DA model and $P$ values $(P<0.05)$ from the $t$-test for further identification of distinct metabolites and relative metabolic pathway analysis. The distinct metabolites were identified by matching accurate molecular weight and characteristic tandem mass spectrometry (MS/MS) fragmentation to the Human Metabolome Database (HMDB, Version 4.0) [31]. If three or more than three MS/MS fragments of a metabolite were matched to spectrum view in the HMDB, we thought that this metabolite was a potential distinct metabolite. All metabolites included in statistical analyses were confirmed by the HMDB with the parameters set as follows: adducts were $M+\mathrm{H}^{+}$and $M+\mathrm{Na}^{+}$for $\mathrm{ESI}^{+}$and $\left.\mathrm{M}-\mathrm{H}\right\rceil^{-}$and $\mathrm{M}+\mathrm{FA}-\mathrm{H}^{-}$for $\mathrm{ESI}^{-}$. The tolerance of mass was set as $10 \mathrm{ppm}$. Some metabolites were further demonstrated by referring to the chemical standards. The identification and comparison of some metabolites against the chemical standard samples were performed according to the retention time, the accurate molecular weight, and the characteristic MS/MS fragments. Then, the receiver-operating characteristic (ROC) curve was used to estimate the accuracy of identified metabolites as potential biomarkers. The differential metabolites with area under the curve (AUC) $>0.9$ were considered potential biomarkers because of high sensitivity and specialty. Metabolic pathway analysis based on the identified distinct metabolites was performed by MetaboAnalyst 4.0 [33], which could get important metabolic pathways by setting the impact-value threshold above 0.10 . The metabolic network analysis was performed with the Cytoscape software (v3.6.1) based on the data from the Kyoto Encyclopedia of Genes and Genomes (KEGG; updated on January 1, 2019) database [34]. The heatmap of all distinct metabolites identified in the plasma and urine samples came from R's ComplexHeatmap (v1.19.1) package.

2.8. Statistical Analysis. Hemorheological data were expressed as mean values \pm standard deviation (SD). Multiple comparisons among groups were performed by oneway analysis of variance (ANOVA) by using the GraphPad 6.0 software (GraphPad Software Inc., San Diego, CA, USA).
Data normality was evaluated by the Kolmogorov-Smirnov test. Statistical homogeneity of variance was estimated by using the F-test. Comparison between two groups was performed by the $t$-test. In the case of homogeneity of variance, $P$ value was calculated using Student's $t$ test; otherwise, $P$ value was calculated by Welch's $t$-test. Nonnormal data were analysed by the Wilcoxon rank-sum test. All the statistical analyses about the identification of distinct metabolites were performed with $\mathrm{R}$ (v3.3.3). $P<0.05$ indicated differences had statistical significance.

\section{Results}

3.1. Effects of RWCI on Hemorheology and Inflammation in Rats with BSS. Compared with healthy rats, BSS rats gradually moved slowly in action. The colors of lips, claws, and eyes were dark red in BSS model rats, which were significantly improved after RWCI and CDST treatment. In addition, the bodies of BSS rats were longer than those of healthy rats. Compared with the control group, both whole blood viscosity and plasma viscosity were significantly increased in the model group $(P<0.01)$ (see Table 1 ), which indicated the BSS model was successfully established and abnormal blood flow had appeared in BSS rats. However, these results were significantly decreased in RWCI and CDST treatment groups compared with the model group $(P<0.05)$, which showed RWCI had a potential to inhibit and improve the hemorheological disorder, thereby preventing the occurrence of BSS.

In addition to hemorheological parameters, the effects of inflammatory factors, including TNF- $\alpha$ and IL-6, on the BSS rats were also evaluated in the present study (see Figure 1). Compared to the control group, the levels of TNF- $\alpha$ and IL- 6 were significantly increased $(P<0.001)$ in BSS model rats. The abnormal levels of cytokines were markedly improved in high-dose and middle-dose RWCI and CDST treatment groups $(P<0.05)$. However, the low dose of RWCI showed no significant effect with only the decreased trend in rats with BSS. The results indicated that BSS might also have a great relationship with inflammation.

3.2. Multivariate Analysis Based on Metabolomics Data of Plasma and Urine Samples. The validation of the systematic method was evaluated by calculating the relative standard deviation (RSD) of retention times and $\mathrm{m} / z$ for repeatability and intermediate precision in $\mathrm{ESI}^{+}$and $\mathrm{ESI}^{-}$modes of plasma and urine samples, respectively. All RSD values were less than $3.3 \%$, indicating this method could be used for subsequent analysis (see Table S1 and Table S2) [35]. The PCA plots of plasma and urine samples in $\mathrm{ESI}^{+}$and $\mathrm{ESI}^{-}$ modes showed significant separations in the model group and other groups. The model was reliable because Q2 was over 0.4 except in PCA of the plasma sample in the ESI mode [36]. Moreover, high-dose RWCI group and CDST group were closer to the control group than middle-dose and low-dose RWCI groups (see Figure S1), manifesting rats in RWCI and CDST treatment groups had similar metabolic patterns with healthy rats. Moreover, the RWCI curative 
TABLE 1: Effects of RWCI on whole blood viscosity and plasma viscosity in rats with BSS.

\begin{tabular}{|c|c|c|c|c|}
\hline \multirow{2}{*}{ Group } & \multicolumn{3}{|c|}{ Whole blood viscosity (mPa.s) } & \multirow{2}{*}{$\begin{array}{c}\text { Plasma viscosity }(\mathrm{mPa} \cdot \mathrm{s}) \\
120 \mathrm{~s}^{-1}\end{array}$} \\
\hline & $10 \mathrm{~s}^{-1}$ & $60 \mathrm{~s}^{-1}$ & $120 \mathrm{~s}^{-1}$ & \\
\hline $\mathrm{C}$ & $11.51 \pm 0.97^{* *}$ & $7.14 \pm 0.74^{* *}$ & $5.95 \pm 0.78^{* *}$ & $1.06 \pm 0.32^{* *}$ \\
\hline $\mathrm{M}$ & $15.38 \pm 1.22$ & $9.02 \pm 0.68$ & $7.39 \pm 0.72$ & $1.42 \pm 0.17$ \\
\hline $\mathrm{TH}$ & $12.03 \pm 1.11^{* *}$ & $7.63 \pm 0.88^{* *}$ & $6.66 \pm 0.69^{*}$ & $1.19 \pm 0.6^{* *}$ \\
\hline TM & $12.77 \pm 1.21^{* *}$ & $7.72 \pm 0.65^{* *}$ & $6.69 \pm 0.67^{*}$ & $1.26 \pm 0.54^{* *}$ \\
\hline $\mathrm{TL}$ & $13.25 \pm 0.82^{* *}$ & $8.15 \pm 0.76^{* *}$ & $7.01 \pm 0.63^{*}$ & $1.39 \pm 0.37^{*}$ \\
\hline CDST & $11.84 \pm 1.34^{* *}$ & $7.43 \pm 0.79^{* *}$ & $6.54 \pm 0.63^{* *}$ & $1.13 \pm 0.60^{* *}$ \\
\hline
\end{tabular}

${ }^{*} P<0.05$ and ${ }^{* *} P<0.01$ vs. model group. C: control group; M: model group; TH: high-dose RWCI treatment group; TM: middle-dose RWCI treatment group; TL: low-dose RWCI treatment group; CDST: compound danshen tablet group.

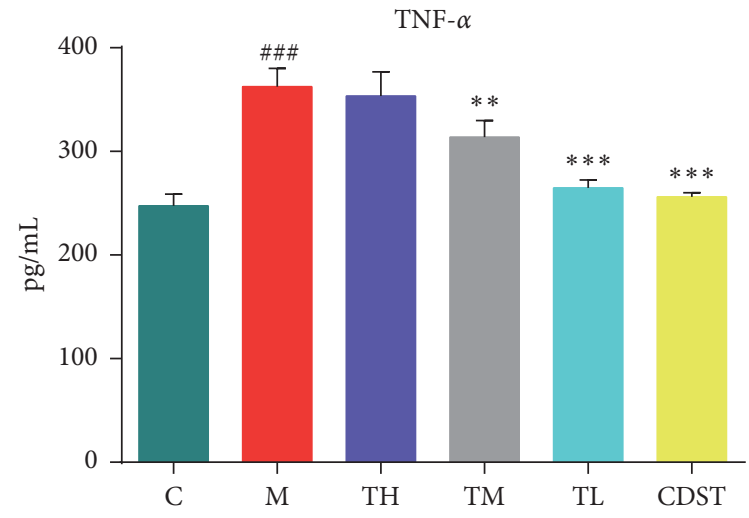

(a)

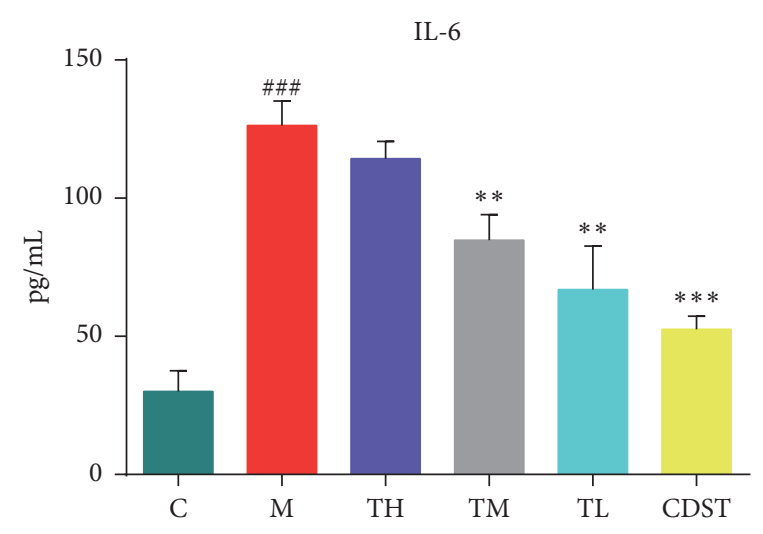

(b)

FIGURE 1: Effects of RWCI on the levels of inflammatory factors (TNF- $\alpha$ and IL-6) in BSS rats. C: control group; M: model group; TH: highdose RWCI treatment group; TM: middle-dose RWCI treatment group; TL: low-dose RWCI treatment group; CDST: compound danshen tablet group. Compared with the control group, \#\#, \#\#, and \# represent $P<0.001, P<0.01$, and $P<0.05$, respectively. Compared with the model group, ${ }^{* * *},{ }^{* *}$, and ${ }^{*}$ represent $P<0.001, P<0.01$, and $P<0.05$, respectively.

effect displayed dosage dependence. OPLS-DA, a supervised clustering model, was performed to find discriminatory metabolic markers associated with BSS. The plasma and urine samples between the control group and the RWCI treatment groups, between the model group and the control group, and between the model group and the RWCI treatment groups had significant separations in OPLS-DA plots in ESI ${ }^{+}$and ESI ${ }^{-}$modes. Permutation tests $(n=200)$ were used to validate the OPLS-DA model (see Figures 2 and 3). All R2 and Q2 values from tests were less than original data, and the intersection of blue regression lines of Q2 points and Y-axis was less than zero, which showed the validity and reliability of the model for further analysis (see Table S3).

\subsection{Identification of Differential Metabolites in Plasma and} Urine Samples. Fourteen and 33 differential metabolites significantly contributed to the occurrence of BSS found in plasma and urine samples, respectively (see Tables 2 and 3 ). The MS/MS spectrum of discriminatory metabolites is shown in Figures S2-S16 (plasma samples) and Figures S17-S51 (urine samples). Moreover, linoleic acid, alpha-linolenic acid, all-trans-retinoic acid, 9-cis-retinoic acid, arachidonic acid, and leukotriene a4 were identified in both plasma and urine samples. Potential distinct metabolites identified in plasma and urine samples were marked in OPLS-DA S-plots in the form of exact mass (see Figures 4 and 5). The ROC curve was used to estimate the accuracy of identified metabolites as potential biomarkers. In plasma samples, expect cortexolone and PC (14:0/20:3), other 12 differential metabolites with AUC $>0.9$ were considered potential biomarkers because of high sensitivity and specialty (see Figure S52). Similarly, in urine samples, 26 differential metabolites with AUC $>0.9$ were considered potential biomarkers (see Figure S53).

In plasma and urine samples, retinoic acids including alltrans-retinoic acid and 9-cis-retinoic acid were identified by matching the retention time, the accurate molecular weight, and the characteristic MS/MS fragments to chemical standard spectra (see Figures S4, S5, S26, and S27). In urine samples, HPETEs including 12(s)-HPETE and 5-HPETE also were identified by comparing with chemical standard spectra (see Figures S48 and S49).

The relative levels of differential metabolites identified from plasma and urine samples are shown in Table 4 . The levels of P1-4 and P11-13 were decreased in plasma samples of BSS rats; however, the levels of P5-10 and P14 were increased. Similarly, the levels of U4, U8-10, U15, U16, and U33 were decreased in urine samples of BSS rats; however, the levels of U1-3, U5-7, U11-14, and U17-32 were increased. The post hoc analysis for multiple comparisons was 


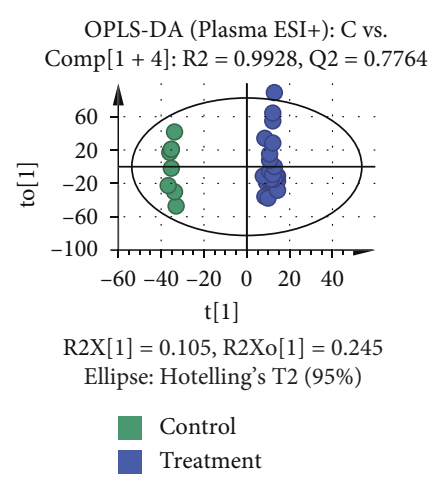

(a)

OPLS-DA (Plasma ESI+): $\mathrm{M}$ vs. C Comp $[1+3] ; \mathrm{R} 2=0.9951, \mathrm{Q} 2=0.8583$

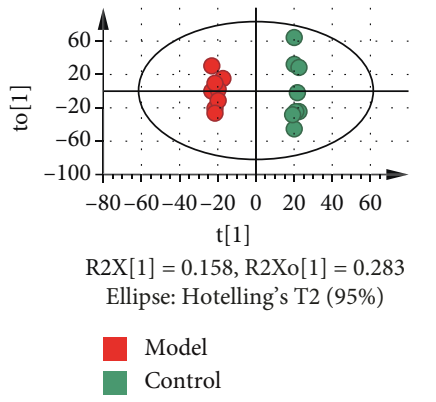

(e)

OPLS-DA (Plasma ESI+): M vs. T Comp $[1+5]: \mathrm{R} 2=0.9950, \mathrm{Q} 2=0.8102$

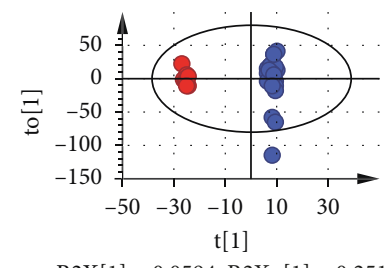

$\mathrm{R} 2 \mathrm{X}[1]=0.0594, \mathrm{R} 2 \mathrm{Xo}[1]=0.251$

Ellipse: Hotelling's T2 (95\%)

$$
\begin{array}{ll}
\text { Model } & \mathrm{R} 2 \\
\text { Treatment } & \mathrm{Q} 2
\end{array}
$$

OPLS-DA (Plasma ESI+): C vs. T

$\mathrm{R} 2=(0.0,0.903), \mathrm{Q} 2=(0.0,-0.611)$

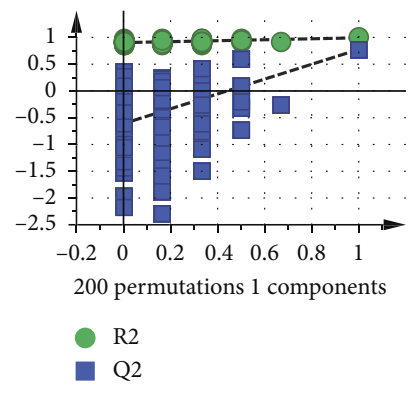

(b)

OPLS-DA (Plasma ESI+): M vs. C $\mathrm{R} 2=(0.0,0.973), \mathrm{Q} 2=(0.0,-0.277)$

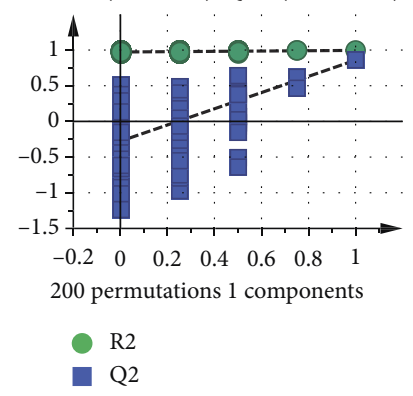

(f)

OPLS-DA (Plasma ESI+): M vs. T $\mathrm{R} 2=(0.0,0.963), \mathrm{Q} 2=(0.0,-0.574)$

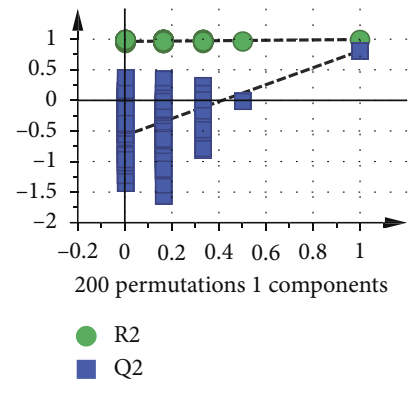

(j)
OPLS-DA (Plasma ESI-): C vs.T

Comp $[1+4]:$ R2 $=0.9928$, Q2 $=0.9004$

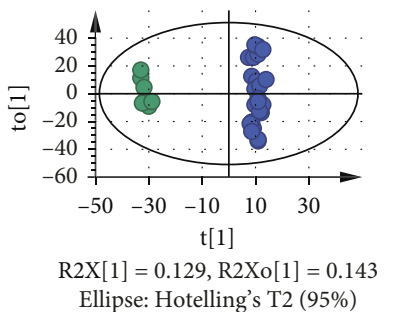

Control

Treatment

(c)

OPLS-DA (Plasma ESI-): M vs. C Comp $[1+3]: \mathrm{R} 2=0.9963, \mathrm{Q} 2=0.9313$

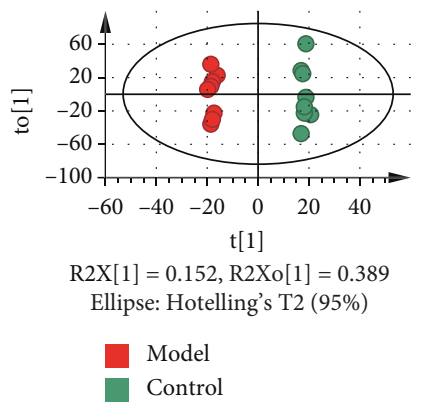

(g)

OPLS-DA (Plasma ESI-): M vs. T Comp $[1+1] \mathrm{R} 2=0.6837 \mathrm{Q} 2=0.3920$

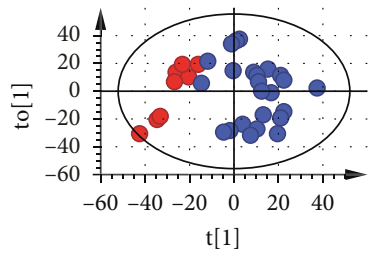

$\mathrm{R} 2 \mathrm{X}[1]=0.186 \mathrm{R} 2 \mathrm{Xo}[1]=0.214$

Ellipse: Hotelling's T2 (95\%)

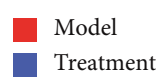

(k)
OPLS-DA (Plasma ESI-): C vs. T $\mathrm{R} 2=(0.0,0.909), \mathrm{Q} 2=(0.0,-0.641)$

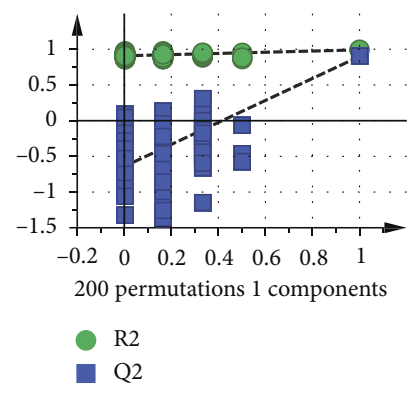

(d)

OPLS-DA (Plasma ESI-): M vs. C $\mathrm{R} 2=(0.0,0.937), \mathrm{Q} 2=(0.0,-0.458)$

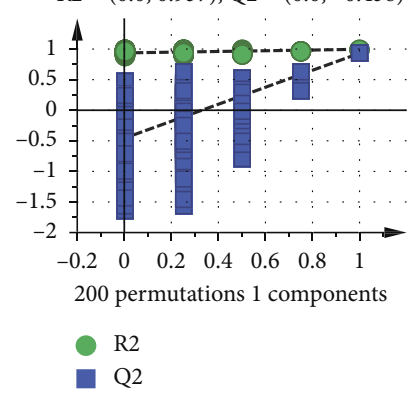

(h)

OPLS-DA (Plasma ESI-): $\mathrm{M}$ vs. T $\mathrm{R} 2=(0.0,0.555), \mathrm{Q} 2=(0.0,-0.425)$

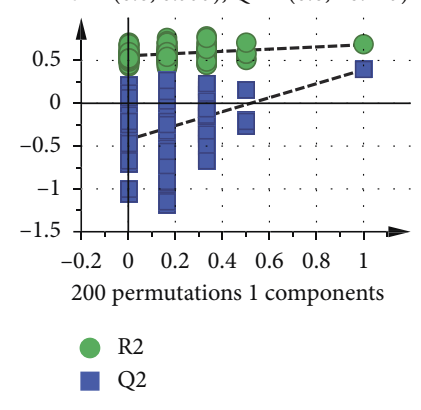

(1)

FIGURE 2: OPLS-DA score plots and permutation test results of metabolomics analysis based on plasma samples: results between the control group and the RWCI treatment groups in the $\mathrm{ESI}^{+}$mode $(\mathrm{a}, \mathrm{b})$ and in the $\mathrm{ESI}^{-}$mode $(\mathrm{c}, \mathrm{d})$; results between the model group and the control group in the $\mathrm{ESI}^{+}$mode $(\mathrm{e}, \mathrm{f})$ and in the $\mathrm{ESI}^{-}$mode $(\mathrm{g}, \mathrm{h})$; results between the model group and the RWCI treatment groups in the ESI ${ }^{+}$mode $(i, j)$ and in the ESI ${ }^{-}$mode $(k, 1)$. All $P$ values were less than 0.01 , calculated by the CV-ANOVA test.

performed for pairwise comparison between the model group and the control group and between the model group and the treatment groups (see Table S4). The levels of distinct metabolites had a very significant difference between the model group and the control group $(P<0.01)$, between the model group and the high-dose RWCI treatment group $(P<0.01)$, and between the model group and the CDST treatment group $(P<0.01)$. All distinct metabolites levels were significantly different between the model group and the middle-dose and low-dose RWCI treatment groups $(P<0.05)$. In contrast, there was no significant difference between the control group and the high-dose RWCI treatment group $(P>0.05)$. RWCI had a potential to alleviate the disordered levels of these metabolites. In addition, the potential distinct metabolites were visually presented using heatmaps. In the heatmap of plasma samples, all samples were clustered as two groups, which indicated the levels of metabolites had a small difference in the control group and treatment groups (see Figure 6). However, all samples were clustered as three groups in the heatmap of urine samples, including the control group, model group, and treatment groups (see Figure 7).

3.4. Relative Metabolic Pathway Analysis. The disturbed metabolic pathways associated with the occurrence of BSS were analysed in the present study. 7 perturbed metabolic pathways were identified in plasma samples, including 


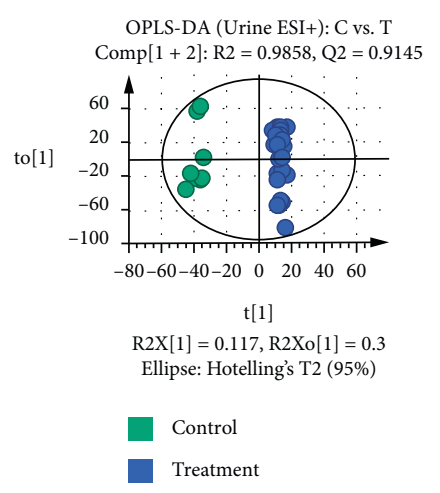

(a)

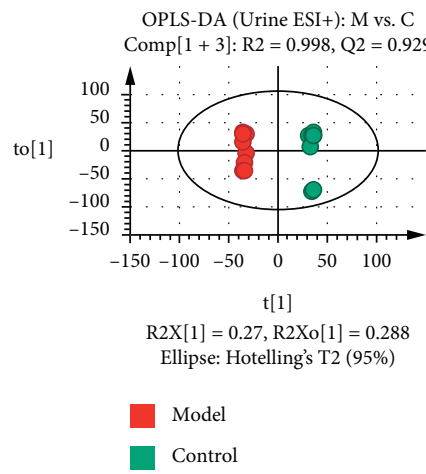

(e)

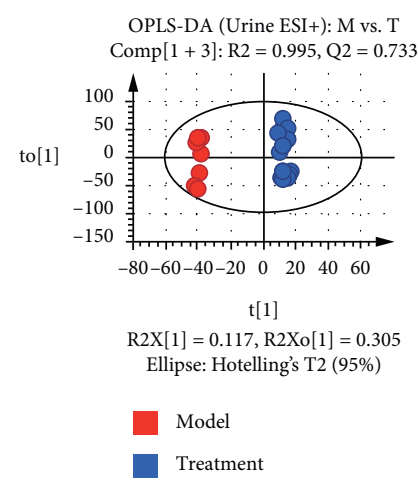

(i)

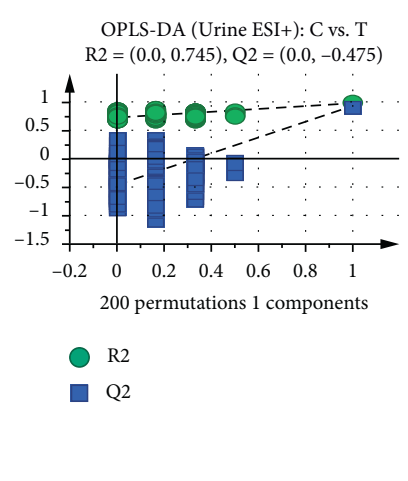

(b)

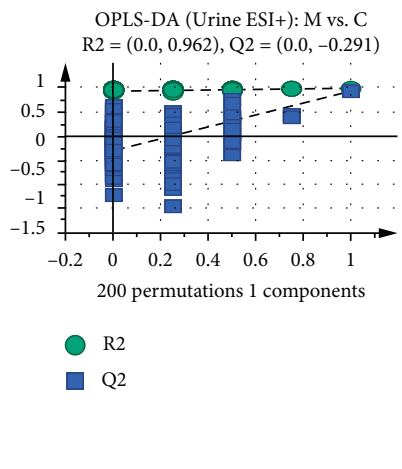

(f)

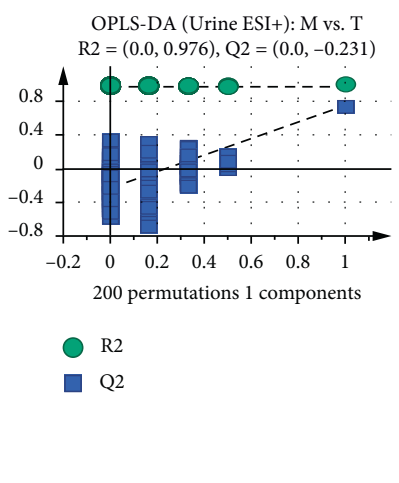

(j)

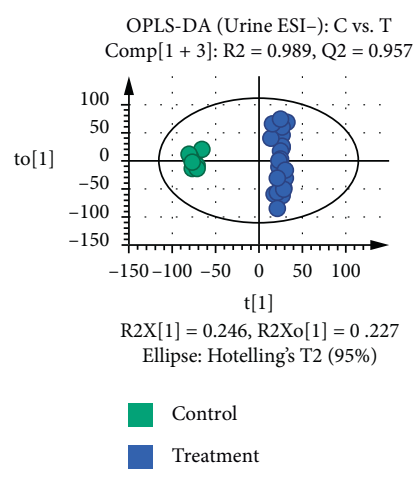

(c)

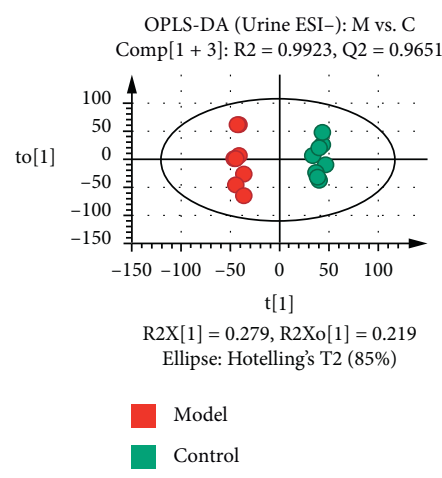

(g)

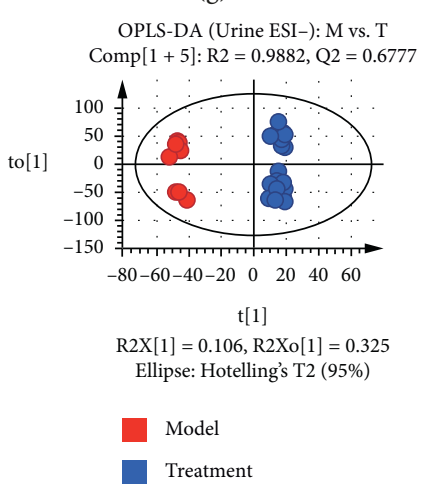

(k)

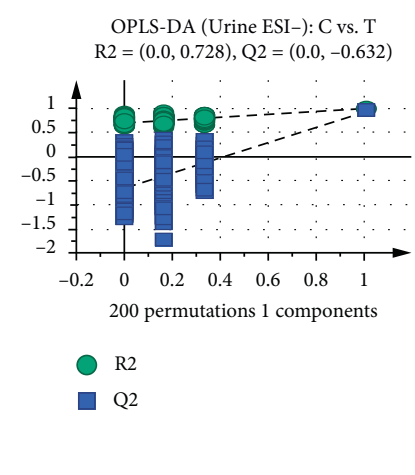

(d)

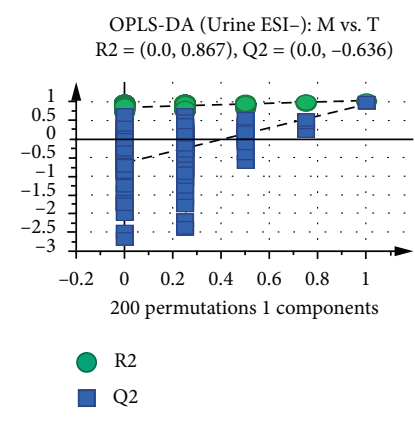

(h)

OPLS-DA (Urine ESI-): M vs. T $\mathrm{R} 2=(0.0,0.936), \mathrm{Q} 2=(0.0,-0.541)$

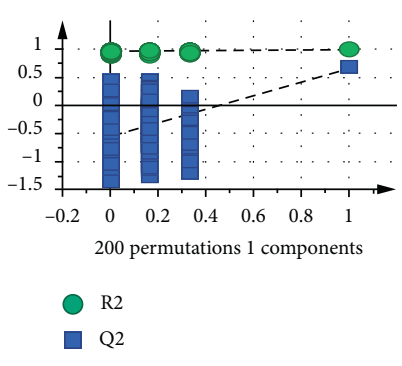

(1)

FIGURE 3: OPLS-DA score plots and permutation test results of metabolomics analysis based on urine samples: results between the control group and the RWCI treatment groups in the $\mathrm{ESI}^{+}$mode $(\mathrm{a}, \mathrm{b})$ and in the $\mathrm{ESI}^{-}$mode $(\mathrm{c}, \mathrm{d})$; results between the model group and the control group in the $\mathrm{ESI}^{+}$mode $(\mathrm{e}, \mathrm{f})$ and in the $\mathrm{ESI}^{-}$mode $(\mathrm{g}, \mathrm{h})$; results between the model group and the RWCI treatment groups in the ESI ${ }^{+}$mode $(i, j)$ and in the ESI $^{-}$mode $(k, 1)$. All $P$ values were less than 0.01 , calculated by the CV-ANOVA test.

steroid hormone biosynthesis, linoleic acid metabolism, alphalinolenic acid metabolism, retinol metabolism, arachidonic acid metabolism, biosynthesis of unsaturated fatty acids, and glycerophospholipid metabolism (see Figure 8(a)). 8 perturbed metabolic pathways were investigated in urine samples, including steroid hormone biosynthesis, linoleic acid metabolism, alpha-linolenic acid metabolism, retinol metabolism, arachidonic acid metabolism, tryptophan metabolism, biosynthesis of unsaturated fatty acids, and steroid biosynthesis (see Figure 8(b)). Therefore, 9 perturbed metabolic pathways (steroid hormone biosynthesis, linoleic acid metabolism, alphalinolenic acid metabolism, retinol metabolism, arachidonic acid metabolism, tryptophan metabolism, biosynthesis of unsaturated fatty acids, steroid biosynthesis, and glycerophospholipid metabolism) were potentially related with the pathogenesis of BSS's development and the pharmacological mechanism of RWCI intervention (see Figure $8(\mathrm{c})$ ). In Figure 8, the pathway impact value represents the significance of the metabolic pathway. The $-\log (P)$ value represents the importance of the metabolic pathway enrichment analysis. The size of the circle is positively correlated with the values of pathway impact and $-\log (P)$. The detailed information of disordered metabolic pathways is presented in Table S5. In this study, the pathway impact value and $P$ value were set to 0.10 and 0.01 according to previous researches $[31,37,38]$. 5 of the 
TABLe 2: Differential metabolites identified in plasma samples.

\begin{tabular}{|c|c|c|c|c|c|c|c|c|c|c|}
\hline No. & $\begin{array}{l}\text { Compound } \\
\text { name }\end{array}$ & Formula & $\begin{array}{c}\text { MS } \\
\text { fragmentation }^{\mathrm{E}}\end{array}$ & $\begin{array}{c}t_{\mathrm{R}} \\
(\mathrm{min})\end{array}$ & $\begin{array}{l}\text { Mass } \\
(\mathrm{Da})\end{array}$ & $\begin{array}{l}\text { KEGG } \\
\text { ID }\end{array}$ & VIP & ESI & $\begin{array}{l}\text { Error } \\
(\mathrm{ppm})\end{array}$ & Pathway \\
\hline P1 & Linoleic acid & $\mathrm{C}_{18} \mathrm{H}_{32} \mathrm{O}_{2}$ & $\begin{array}{c}91.0550,119.0844 \\
121.099\end{array}$ & 23.98 & 279.2357 & C01595 & 1.13 & - & 10 & Linoleic acid \\
\hline P2 & $\begin{array}{c}\text { Alpha- } \\
\text { linolenic acid }\end{array}$ & $\mathrm{C}_{18} \mathrm{H}_{30} \mathrm{O}_{2}$ & $\begin{array}{c}91.0550,119.0844 \\
121.0991\end{array}$ & 21.6 & 279.2301 & $\mathrm{C} 06427$ & 1.42 & + & 2 & Alpha-linolenic acid \\
\hline P3 & Retinoic acids & $\mathrm{C}_{20} \mathrm{H}_{28} \mathrm{O}_{2}$ & $\begin{array}{l}123.0932 \\
149.1257 \\
301.2041\end{array}$ & 14.34 & 301.2144 & C00777 & 3.86 & + & 6 & Retinol \\
\hline $\mathrm{P} 4$ & Retinyl ester & $\mathrm{C}_{20} \mathrm{H}_{30} \mathrm{O}_{2}$ & $\begin{array}{l}134.8988 \\
146.9695 \\
283.2684\end{array}$ & 21.42 & 301.2196 & $\mathrm{C} 02075$ & 1.14 & - & 8 & Retinol \\
\hline P5 & $\begin{array}{l}\text { Arachidonic } \\
\text { acid }\end{array}$ & $\mathrm{C}_{20} \mathrm{H}_{32} \mathrm{O}_{2}$ & $\begin{array}{l}106.9040 \\
162.8759 \\
190.8695\end{array}$ & 22.9 & 305.2454 & C00219 & 3.63 & + & 7 & Arachidonic acid \\
\hline P6 & Leukotriene a4 & $\mathrm{C}_{20} \mathrm{H}_{30} \mathrm{O}_{3}$ & $\begin{array}{l}243.9020 \\
245.8972 \\
317.2140\end{array}$ & 15.37 & 317.2149 & C00909 & 1.35 & - & 8 & Arachidonic acid \\
\hline P7 & Cortexolone & $\mathrm{C}_{21} \mathrm{H}_{30} \mathrm{O}_{4}$ & $\begin{array}{l}121.0644 \\
123.0799 \\
171.1152\end{array}$ & 9.76 & 347.2197 & $\mathrm{C} 05488$ & 2.45 & + & 6 & Steroid hormone biosynthesis \\
\hline P8 & $\begin{array}{c}21- \\
\text { Deoxycortisol }\end{array}$ & $\mathrm{C}_{21} \mathrm{H}_{30} \mathrm{O}_{4}$ & $\begin{array}{l}134.8972 \\
243.8986 \\
245.8962\end{array}$ & 9.81 & 391.2133 & C05497 & 2.27 & - & 2 & Steroid hormone biosynthesis \\
\hline P9 & $\begin{array}{l}\text { Cholesterol } \\
\text { sulfate }\end{array}$ & $\mathrm{C}_{27} \mathrm{H}_{46} \mathrm{O}_{4} \mathrm{~S}$ & $\begin{array}{l}112.9903 \\
327.2350 \\
383.1917\end{array}$ & 27.57 & 465.3014 & C18043 & 1.13 & - & 6 & Steroid hormone biosynthesis \\
\hline $\mathrm{P} 10$ & PC (15:0/18:2) & $\mathrm{C}_{41} \mathrm{H}_{78} \mathrm{NO}_{8} \mathrm{P}$ & $\begin{array}{l}122.9904 \\
279.2365 \\
393.2784\end{array}$ & 25.8 & 788.5378 & $\mathrm{C} 00157$ & 1.04 & - & 9 & \\
\hline P11 & PC (14:0/20:3) & $\mathrm{C}_{42} \mathrm{H}_{78} \mathrm{NO}_{8} \mathrm{P}$ & $\begin{array}{l}183.0163 \\
259.2466 \\
303.2379\end{array}$ & 27.99 & 800.5378 & $\mathrm{C} 00157$ & 1.75 & - & 9 & \\
\hline $\mathrm{P} 12$ & PC (14:0/20:1) & $\mathrm{C}_{42} \mathrm{H}_{82} \mathrm{NO}_{8} \mathrm{P}$ & $\begin{array}{l}112.9912 \\
183.0157 \\
305.2471\end{array}$ & 27.1 & 804.5719 & $\mathrm{C} 00157$ & 1.17 & - & 5 & $\begin{array}{l}\text { Arachidonic acid, alpha- } \\
\text { linolenic acid, linoleic acid, and } \\
\text { glycerophospholipid }\end{array}$ \\
\hline P13 & PC (16:0/20:4) & $\mathrm{C}_{44} \mathrm{H}_{80} \mathrm{NO}_{8} \mathrm{P}$ & $\begin{array}{l}239.0619 \\
255.2331 \\
303.2323\end{array}$ & 28.1 & 826.5543 & $\mathrm{C} 00157$ & 1.71 & - & 7 & \\
\hline P14 & PC $(20: 4 / 18: 0)$ & $\mathrm{C}_{46} \mathrm{H}_{84} \mathrm{NO}_{8} \mathrm{P}$ & $\begin{array}{l}255.2368 \\
279.2371 \\
303.2366\end{array}$ & 25.4 & 854.584 & $\mathrm{C} 00157$ & 1.15 & - & 9 & \\
\hline
\end{tabular}

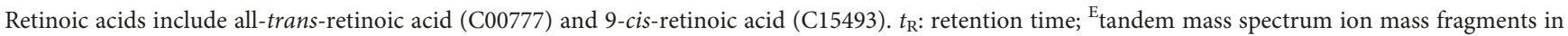
MS/MS: three major fragment ions with high abundances of each distinct metabolite are listed; ESI ${ }^{+}$Q/TOF mass spectrometer with an electrospray ionization in the positive mode; $\mathrm{ESI}^{-}$: Q/TOF mass spectrometer with an electrospray ionization in the negative mode; PC: phosphatidylcholine. PCs are involved in arachidonic acid metabolism, alpha-linolenic acid metabolism, linoleic acid metabolism, and glycerophospholipid metabolism.

metabolic pathways (steroid hormone biosynthesis, linoleic acid metabolism, alpha-linolenic acid metabolism, retinol metabolism, and arachidonic acid metabolism) showed evident changes in BSS rats according to impact $>0.1$ and $P<0.01$, which indicated they might be significantly associated with BSS's development and RWCI intervention. The glycerophospholipid metabolism pathway had a potential relativity because impact $>0.1$ but $P>0.05$.

A metabolic network of all differential metabolites identified in plasma and urine samples is displayed in Figure 9. 9 perturbed metabolic pathways are presented in different colors. Retinol metabolism was not involved in the formation of the whole network.

\section{Discussion}

In recent years, the UPLC/MS technique has been widely used to explore the pathological mechanism of diseases and the pharmacological activity of medicines by identifying the distinct metabolites. For example, UPLC/MS-based metabolomics methods were used to identify the differential metabolites in the rat model of postmenopausal osteoporosis, thereby illustrating its pathogenesis and the therapeutic effects of Acanthopanax senticosus [39]. Furthermore, a UPLC/MSbased metabolomics study found that the antiulcer activity of C. alternifolius tubers might be related to $\alpha$-carbonic anhydrase inhibitory, anti-inflammatory, and analgesic activity by their antioxidant activity and downregulation of several 
Table 3: Differential metabolites identified in urine samples.

\begin{tabular}{|c|c|c|c|c|c|c|c|c|c|c|}
\hline No. & Compound name & Formula & $\begin{array}{c}\text { MS } \\
\text { fragmentation }^{\mathrm{E}}\end{array}$ & $\begin{array}{c}t_{\mathrm{R}} \\
(\mathrm{min})\end{array}$ & $\begin{array}{l}\text { Mass } \\
(\mathrm{Da})\end{array}$ & $\begin{array}{l}\text { KEGG } \\
\text { ID }\end{array}$ & VIP & ESI & $\begin{array}{l}\text { Error } \\
(\mathrm{ppm})\end{array}$ & Pathway \\
\hline U1 & L-Kynurenine & $\mathrm{C}_{10} \mathrm{H}_{12} \mathrm{~N}_{2} \mathrm{O}_{3}$ & $\begin{array}{l}146.0583 \\
147.1145 \\
165.0665\end{array}$ & 6.95 & 231.074 & $\mathrm{C} 00328$ & 1.78 & + & 0 & $\begin{array}{l}\text { Tryptophan } \\
\text { metabolism }\end{array}$ \\
\hline $\mathrm{U} 2$ & $\begin{array}{l}\text { 4-(2-Aminophenyl)-2,4- } \\
\text { dioxobutanoic acid }\end{array}$ & $\mathrm{C}_{10} \mathrm{H}_{9} \mathrm{NO}_{4}$ & $\begin{array}{l}144.0485 \\
160.0436 \\
165.0595\end{array}$ & 0.67 & 252.0532 & $\mathrm{C} 01252$ & 1.9 & - & 7 & $\begin{array}{l}\text { Tryptophan } \\
\text { metabolism }\end{array}$ \\
\hline U3 & Estrone & $\mathrm{C}_{18} \mathrm{H}_{22} \mathrm{O}$ & $\begin{array}{l}295.0858 \\
171.1138 \\
271.1667\end{array}$ & 7.84 & 269.1558 & C00468 & 1.31 & - & 4 & $\begin{array}{l}\text { Steroid hormone } \\
\text { biosynthesis }\end{array}$ \\
\hline U4 & Linoleic acid & $\mathrm{C}_{18} \mathrm{H}_{32} \mathrm{O}$ & $\begin{array}{c}281.0719,97.1016 \\
121.1001\end{array}$ & 24.15 & 281.2455 & C01595 & 1.54 & + & 7 & $\begin{array}{l}\text { Linoleic acid } \\
\text { metabolism }\end{array}$ \\
\hline U5 & Androstanedione & $\mathrm{C}_{19} \mathrm{H}_{28} \mathrm{O}$ & $\begin{array}{l}296.9614 \\
109.1013 \\
149.0218\end{array}$ & 8.99 & 287.1982 & C00674 & 1.77 & + & 8 & $\begin{array}{l}\text { Steroid hormone } \\
\text { biosynthesis }\end{array}$ \\
\hline U6 & Dehydroepiandrosterone & $\mathrm{C}_{19} \mathrm{H}_{28} \mathrm{O}$ & $\begin{array}{c}296.9611 \\
145.0980 \\
173.1301\end{array}$ & 13.35 & 289.2135 & C01227 & 1.21 & + & 9 & $\begin{array}{l}\text { Steroid hormone } \\
\text { biosynthesis }\end{array}$ \\
\hline U7 & Estradiol & $\mathrm{C}_{18} \mathrm{H}_{24} \mathrm{O}_{2}$ & $\begin{array}{l}119.0852 \\
145.0992 \\
243.1364\end{array}$ & 8.15 & 295.1644 & C00951 & 1.19 & + & 8 & $\begin{array}{l}\text { Steroid hormone } \\
\text { biosynthesis }\end{array}$ \\
\hline U8 & Stearidonic acid & $\mathrm{C}_{18} \mathrm{H}_{28} \mathrm{O}_{2}$ & $\begin{array}{l}133.0992 \\
149.0206 \\
159.1133\end{array}$ & 8.01 & 299.1977 & C16300 & 1.09 & + & 1 & $\begin{array}{l}\text { Alpha-linolenic } \\
\text { acid metabolism }\end{array}$ \\
\hline U9 & Alpha-linolenic acid & $\mathrm{C}_{18} \mathrm{H}_{30} \mathrm{O}_{2}$ & $\begin{array}{l}109.1004 \\
119.0844 \\
121.0991\end{array}$ & 10.41 & 301.2138 & $\mathrm{C} 06427$ & 1.01 & + & 0 & $\begin{array}{l}\text { Alpha-linolenic } \\
\text { acid metabolism }\end{array}$ \\
\hline U10 & Retinoic acids & $\mathrm{C}_{20} \mathrm{H}_{28} \mathrm{O}_{2}$ & $\begin{array}{l}123.0932 \\
149.1257 \\
301.2041\end{array}$ & 17.13 & 301.2157 & $\mathrm{C} 00777$ & 1.2 & + & 2 & Retinol metabolism \\
\hline U11 & 2-Hydroxyestradiol & $\mathrm{C}_{18} \mathrm{H}_{24} \mathrm{O}_{3}$ & $\begin{array}{l}135.0426 \\
149.0214 \\
161.0941\end{array}$ & 22.04 & 311.162 & C05301 & 2.83 & + & 1 & $\begin{array}{l}\text { Steroid hormone } \\
\text { biosynthesis }\end{array}$ \\
\hline U12 & Etiocholanedione & $\mathrm{C}_{19} \mathrm{H}_{28} \mathrm{O}_{2}$ & $\begin{array}{c}96.9611,109.1007 \\
175.1449\end{array}$ & 7.44 & 311.1986 & $\mathrm{C} 03772$ & 1.77 & + & 1 & $\begin{array}{l}\text { Steroid hormone } \\
\text { biosynthesis }\end{array}$ \\
\hline U13 & Dihydrotestosterone & $\mathrm{C}_{19} \mathrm{H}_{30} \mathrm{O}_{2}$ & $\begin{array}{l}109.1005 \\
215.0669 \\
227.1756\end{array}$ & 8.99 & 313.2132 & $\mathrm{C} 03917$ & 1.71 & + & 2 & $\begin{array}{l}\text { Steroid hormone } \\
\text { biosynthesis }\end{array}$ \\
\hline U14 & Androsterone & $\mathrm{C}_{19} \mathrm{H}_{30} \mathrm{O}_{2}$ & $\begin{array}{l}107.0849 \\
145.0990 \\
147.1144\end{array}$ & 10.03 & 313.2143 & $\mathrm{C} 00523$ & 1.52 & + & 2 & $\begin{array}{l}\text { Steroid hormone } \\
\text { biosynthesis }\end{array}$ \\
\hline U15 & Hydroxyretinoic acid & $\mathrm{C}_{20} \mathrm{H}_{28} \mathrm{O}_{3}$ & $\begin{array}{l}105.0706 \\
189.0532 \\
215.1055\end{array}$ & 8.4 & 317.2081 & C16677 & 1.33 & + & 0 & Retinol metabolism \\
\hline U16 & $\begin{array}{l}\text { All-trans-5,6-epoxyretinoic } \\
\text { acid }\end{array}$ & $\mathrm{C}_{20} \mathrm{H}_{28} \mathrm{O}$ & $\begin{array}{l}3105.0706 \\
189.0532 \\
215.1055\end{array}$ & 11.1 & 317.211 & C16680 & 1.36 & + & 0 & Retinol metabolism \\
\hline U17 & Deoxycorticosterone & $\mathrm{C}_{21} \mathrm{H}_{30} \mathrm{O}_{3}$ & $\begin{array}{l}217.0565 \\
289.2139 \\
313.2133\end{array}$ & 6.03 & 331.2247 & C03205 & 1.24 & + & 6 & $\begin{array}{l}\text { Steroid hormone } \\
\text { biosynthesis }\end{array}$ \\
\hline $\mathrm{U} 18$ & 17-Hydroxyprogesterone & $\mathrm{C}_{21} \mathrm{H}_{30} \mathrm{O}_{3}$ & $\begin{array}{l}109.1005 \\
271.0565 \\
289.2139\end{array}$ & 11.55 & 331.2251 & $\mathrm{C} 01176$ & 3.56 & + & 5 & $\begin{array}{l}\text { Steroid hormone } \\
\text { biosynthesis }\end{array}$ \\
\hline U19 & 11b-Hydroxyprogesterone & $\mathrm{C}_{20} \mathrm{H}_{28} \mathrm{O}_{4}$ & $\begin{array}{l}269.1883 \\
287.1989 \\
333.2040\end{array}$ & 9.79 & 333.204 & C05498 & 1.83 & + & 6 & $\begin{array}{l}\text { Steroid hormone } \\
\text { biosynthesis }\end{array}$ \\
\hline $\mathrm{U} 20$ & $\begin{array}{l}\text { 17-Alpha,20-alpha } \\
\text { dihydroxypregn-4-en-3-one }\end{array}$ & $\mathrm{C}_{21} \mathrm{H}_{32} \mathrm{O}_{3}$ & $\begin{array}{l}143.0840 \\
177.1606 \\
261.1457\end{array}$ & 7.66 & 333.2395 & $\mathrm{C} 04518$ & 1.05 & + & 9 & $\begin{array}{l}\text { Steroid hormone } \\
\text { biosynthesis }\end{array}$ \\
\hline
\end{tabular}


TABLE 3: Continued.

\begin{tabular}{|c|c|c|c|c|c|c|c|c|c|c|}
\hline No. & Compound name & Formula & $\begin{array}{c}\text { MS } \\
\text { fragmentation }^{\mathrm{E}} \\
\end{array}$ & $\begin{array}{c}t_{\mathrm{R}} \\
(\mathrm{min})\end{array}$ & $\begin{array}{l}\text { Mass } \\
(\mathrm{Da})\end{array}$ & $\begin{array}{l}\text { KEGG } \\
\text { ID }\end{array}$ & VIP & ESI & $\begin{array}{l}\text { Error } \\
(\mathrm{ppm})\end{array}$ & Pathway \\
\hline $\mathrm{U} 21$ & $\begin{array}{l}\text { 13-1-Hydroperoxylinoleic } \\
\text { acid }\end{array}$ & $\mathrm{C}_{18} \mathrm{H}_{32} \mathrm{O}_{4}$ & $\begin{array}{l}156.8872, \\
163.0721 \\
267.0013\end{array}$ & 13.5 & 335.2196 & $\mathrm{C} 04717$ & 1.5 & + & 1 & $\begin{array}{l}\text { Linoleic acid } \\
\text { metabolism }\end{array}$ \\
\hline $\mathrm{U} 22$ & 12(s)-Leukotriene b4 & $\mathrm{C}_{20} \mathrm{H}_{32} \mathrm{O}_{4}$ & $\begin{array}{l}113.0288 \\
141.8739 \\
247.0743\end{array}$ & 10.41 & 335.226 & C04853 & 1.26 & - & 10 & $\begin{array}{l}\text { Arachidonic acid } \\
\text { metabolism }\end{array}$ \\
\hline $\mathrm{U} 23$ & $\begin{array}{l}\text { 3a,21-Dihydroxy-5b- } \\
\text { pregnane-11,20-dione }\end{array}$ & $\mathrm{C}_{21} \mathrm{H}_{32} \mathrm{O}_{4}$ & $\begin{array}{c}97.0710,313.2144 \\
349.2357\end{array}$ & 11.51 & 349.2352 & C05478 & 2.16 & + & 6 & $\begin{array}{l}\text { Steroid hormone } \\
\text { biosynthesis }\end{array}$ \\
\hline $\mathrm{U} 24$ & $\begin{array}{c}17 \mathrm{a}, 21- \\
\text { Dihydroxypregnenolone }\end{array}$ & $\mathrm{C}_{21} \mathrm{H}_{32} \mathrm{O}_{4}$ & $\begin{array}{l}157.0990 \\
313.2144 \\
349.2357\end{array}$ & 6.19 & 349.2356 & C05487 & 5.12 & + & 5 & $\begin{array}{l}\text { Steroid hormone } \\
\text { biosynthesis }\end{array}$ \\
\hline $\mathrm{U} 25$ & Cortisol & $\mathrm{C}_{21} \mathrm{H}_{30} \mathrm{O}_{5}$ & $\begin{array}{l}329.1620 \\
331.1892 \\
361.2032\end{array}$ & 8.65 & 361.2015 & C00735 & 1.21 & - & 2 & $\begin{array}{l}\text { Steroid hormone } \\
\text { biosynthesis }\end{array}$ \\
\hline U26 & Leukotriene a4 & $\mathrm{C}_{20} \mathrm{H}_{30} \mathrm{O}_{3}$ & $\begin{array}{l}243.1349 \\
245.0459 \\
255.0635\end{array}$ & 9.89 & 363.2199 & C00909 & 1.77 & - & 6 & $\begin{array}{l}\text { Arachidonic acid } \\
\text { metabolism }\end{array}$ \\
\hline $\mathrm{U} 27$ & $\begin{array}{c}\text { 11b,17a,21- } \\
\text { Trihydroxypregnenolone }\end{array}$ & $\mathrm{C}_{21} \mathrm{H}_{32} \mathrm{O}_{5}$ & $\begin{array}{l}\text { 109.0996, } \\
157.0980 \\
242.1904\end{array}$ & 5.01 & 365.2299 & C05489 & 1.81 & + & 6 & $\begin{array}{c}\text { Steroid hormone } \\
\text { biosynthesis }\end{array}$ \\
\hline $\mathrm{U} 28$ & Dihydrocortisol & $\mathrm{C}_{21} \mathrm{H}_{32} \mathrm{O}_{5}$ & $\begin{array}{l}157.0980 \\
329.2062 \\
365.2264\end{array}$ & 9.66 & 365.2302 & C05471 & 1.21 & + & 6 & $\begin{array}{l}\text { Steroid hormone } \\
\text { biosynthesis }\end{array}$ \\
\hline U29 & 15(s)-HETE & $\mathrm{C}_{20} \mathrm{H}_{32} \mathrm{O}_{3}$ & $\begin{array}{l}113.0290 \\
175.0275 \\
245.8979\end{array}$ & 10.53 & 365.2346 & C04742 & 1.3 & - & 3 & $\begin{array}{l}\text { Arachidonic acid } \\
\text { metabolism }\end{array}$ \\
\hline U30 & $\begin{array}{l}\text { 14,15-Epoxy-5,8,11- } \\
\text { eicosatrienoic acid }\end{array}$ & $\mathrm{C}_{20} \mathrm{H}_{32} \mathrm{O}_{3}$ & $\begin{array}{l}113.0293 \\
219.1411 \\
229.0205\end{array}$ & 8.99 & 365.2353 & C14771 & 3.39 & - & 5 & $\begin{array}{l}\text { Arachidonic acid } \\
\text { metabolism }\end{array}$ \\
\hline U31 & HPETEs & $\mathrm{C}_{20} \mathrm{H}_{32} \mathrm{O}_{4}$ & $\begin{array}{l}215.1032 \\
249.0927 \\
335.2313\end{array}$ & 3.87 & 381.2298 & C05965 & 1.79 & - & 4 & $\begin{array}{l}\text { Arachidonic acid } \\
\text { metabolism }\end{array}$ \\
\hline U32 & Cortisone & $\mathrm{C}_{21} \mathrm{H}_{28} \mathrm{O}_{5}$ & $\begin{array}{c}231.1381 \\
259.1132 \\
317.2115\end{array}$ & 9.43 & 405.1908 & C00762 & 1.02 & - & 3 & $\begin{array}{l}\text { Steroid hormone } \\
\text { biosynthesis }\end{array}$ \\
\hline U33 & 4a-Methylfecosterol & $\mathrm{C}_{29} \mathrm{H}_{48} \mathrm{O}$ & $\begin{array}{c}\text { 81.0721, 97.1024 } \\
413.2669\end{array}$ & 25.21 & 435.3581 & C15776 & 1 & + & 4 & $\begin{array}{c}\text { Steroid } \\
\text { biosynthesis }\end{array}$ \\
\hline
\end{tabular}

Retinoic acids include all-trans-retinoic acid (C00777) and 9-cis-retinoic acid (C15493). HPETEs include 12(s)-HPETE (C05965) and 5-HPETE (C05356). $t_{\mathrm{R}}$ : retention time; ${ }^{\mathrm{E}}$ tandem mass spectrum ion mass fragments in MS/MS: three major fragment ions with high abundances of each distinct metabolite are listed; $\mathrm{ESI}^{+}$: Q/TOF mass spectrometer with an electrospray ionization in the positive mode; $\mathrm{ESI}^{-}: \mathrm{Q} / \mathrm{TOF}$ mass spectrometer with an electrospray ionization in the negative mode.

inflammatory mediators [40]. We also explored the inhibitory effect of methotrexate on rheumatoid arthritis inflammation using UPLC-Q/TOF-MS [41]. In addition to investigating pharmacological activity, the UPLC/MS technique was also used in metabolite identification, which might provide new insights for the development and progression of diseases. The metabolite biomarkers, such as aristolochic acid, kynurenic acid, and hippuric acid, might be associated with nephrotoxicity [42-45]. UPLC/MS was also used to analyse and discover reliable biomarkers for the diagnosis and prognosis of hepatitis B-related acute-on-chronic liver failure (ACLF) [46]. Researchers found that 17 metabolites were related to prognosis of hepatitis B-related ACLF.

In the present study, UPLC-Q/TOF-MS-based metabolomics was utilized to identify distinct metabolites associated with the occurrence of BSS and the disturbed metabolic pathways in BSS rats, thereby uncovering its pathogenesis and the therapeutic effect of RWCI against BSS. Hemorheology was employed to explore the flow properties of blood in the vessel [47]. In the current study, whole blood viscosity and plasma viscosity, the main hemorheological parameters, were higher in BSS rats than in healthy control rats. Increased blood viscosity was related to the decrease of blood flow speed and tissue hypoxia, which might further lead to metabolic disorders. However, whole blood viscosity and plasma viscosity were significantly improved in BSS rats after RWCI treatment, indicating RWCI might have a potential role in activating blood circulation and eliminating BSS. In addition, other studies showed that thrombin time, prothrombin time, and activated partial thromboplastin time were significantly shortened and fibrinogen content was significantly increased in BSS rats, 


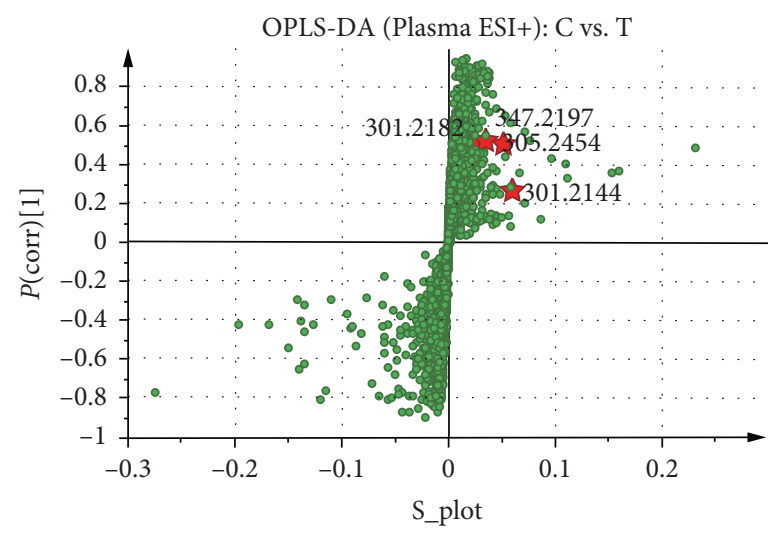

(a)

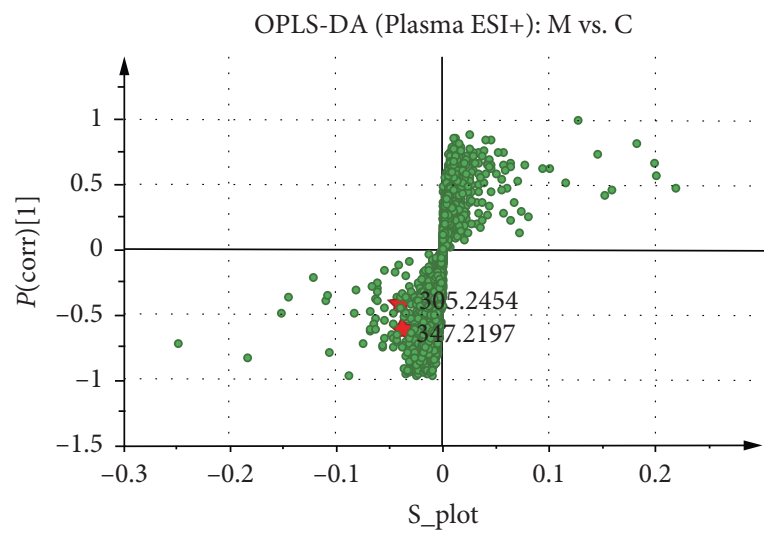

(c)

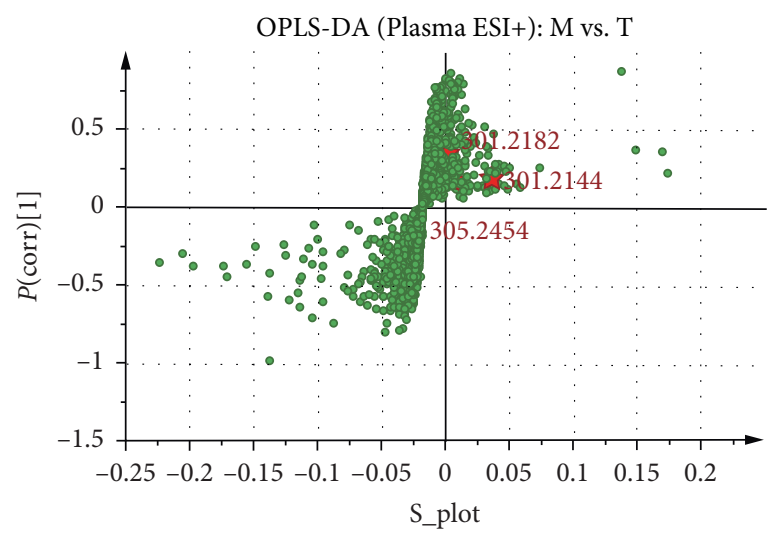

(e)

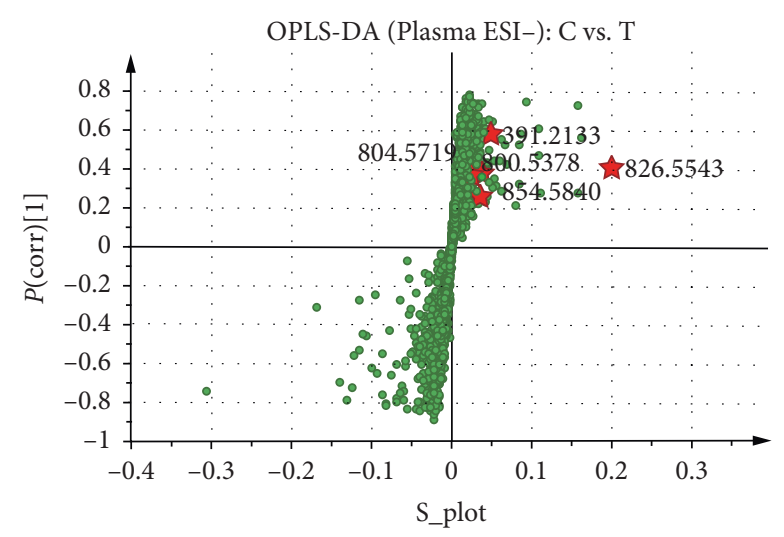

(b)

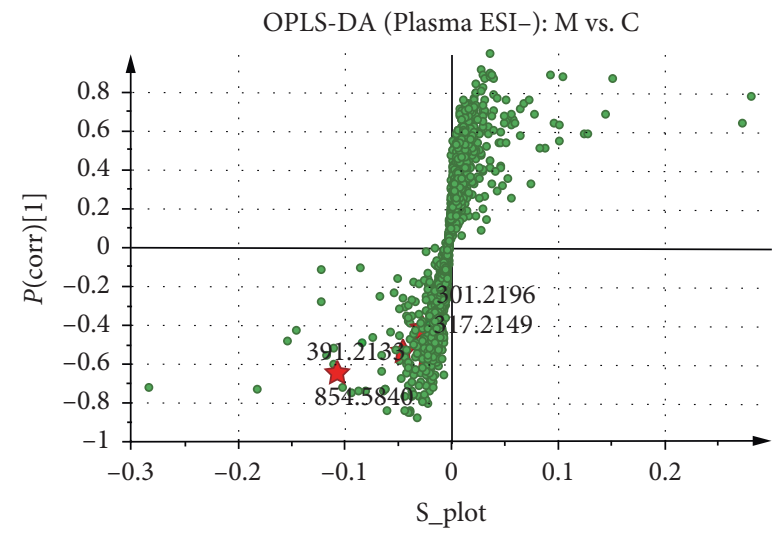

(d)

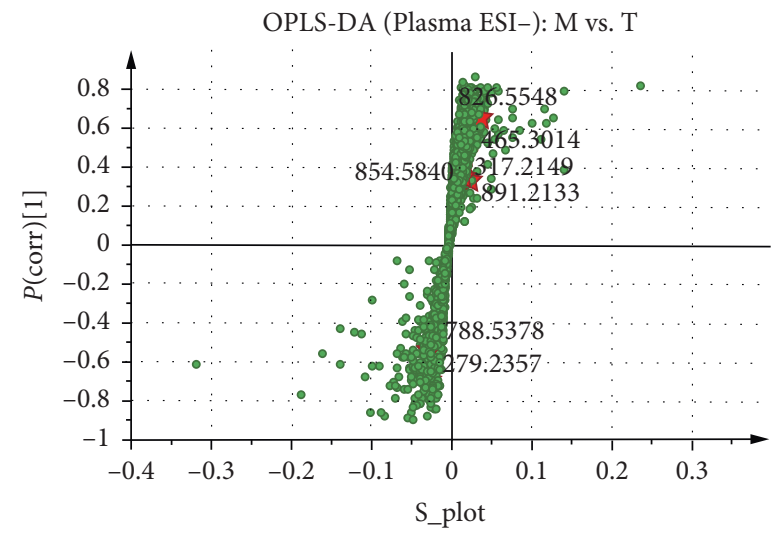

(f)

FIGURE 4: OPLS-DA S-plots of metabolomics analysis based on plasma samples. All differential metabolites are shown in S-plots, including the identified results between the control group and the RWCI treatment groups in the $\mathrm{ESI}^{+}$mode (a) and in the ESI ${ }^{-}$mode (b), the identified results between the model group and the control group in the ESI ${ }^{+}$mode (c) and in the ESI ${ }^{-}$mode (d), and the identified results between the model group and the RWCI treatment groups in the $\mathrm{ESI}^{+}$mode (e) and in the $\mathrm{ESI}^{-}$mode (f). C: control group; M: model group; T: RWCI treatment groups. The $p$ (corr)[1]-axis shows the reliability of each variable in $X$.

which indicated that the blood of rats with BSS is in a state of being easily coagulated $[5,48]$. The coagulation dysfunction is a vital risk factor for thrombosis and cardiovascular diseases [49]. The levels of TNF- $\alpha$ and IL-6 were significantly increased in BSS model rats compared to healthy rats. Moreover, high dose and middle dose of RWCI were effective in the improvement of inflammation in BSS rats. Plasma and urine samples were commonly used for metabolomics analysis. 14 and 33 differential metabolites were identified in plasma and urine samples from BSS rats using metabolomics based on UPLC-Q/TOF-MS, involving 9 disturbed metabolic pathways. 5 metabolic pathways were significantly related to BSS's development and RWCI intervention. The levels of these differential metabolites in BSS rats could be improved after RWCI treatment. 
OPLS-DA (Urine ESI+): C vs. T

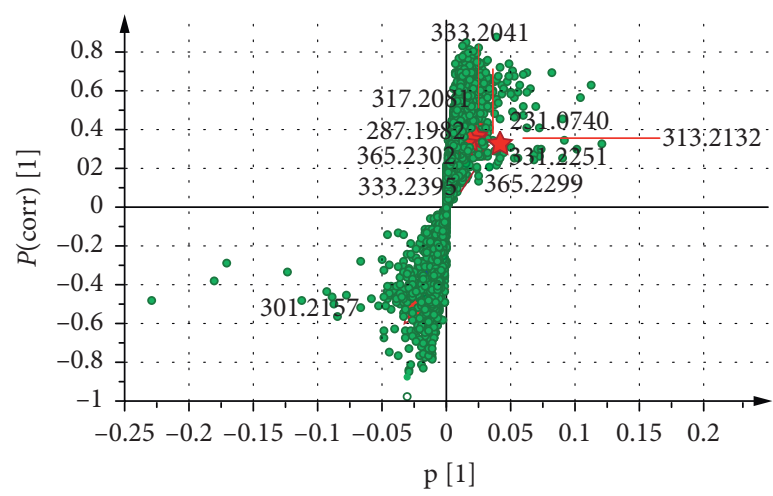

(a)

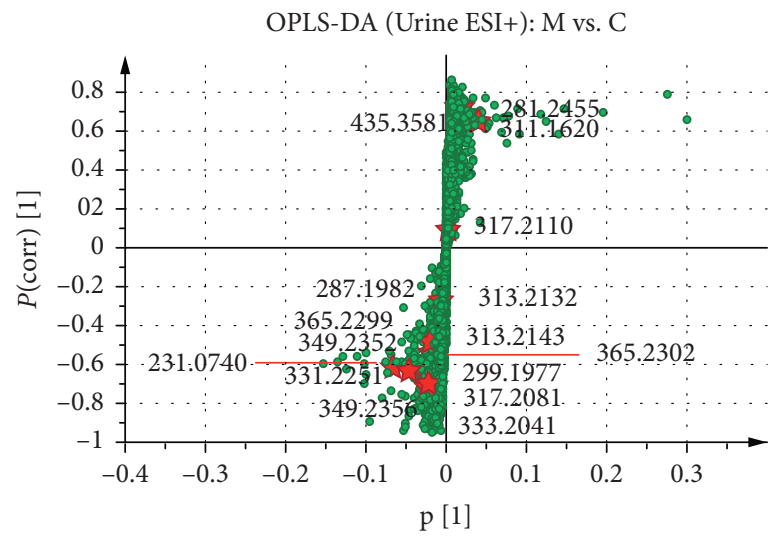

(c)

OPLS-DA (Urine ESI+): M vs. T

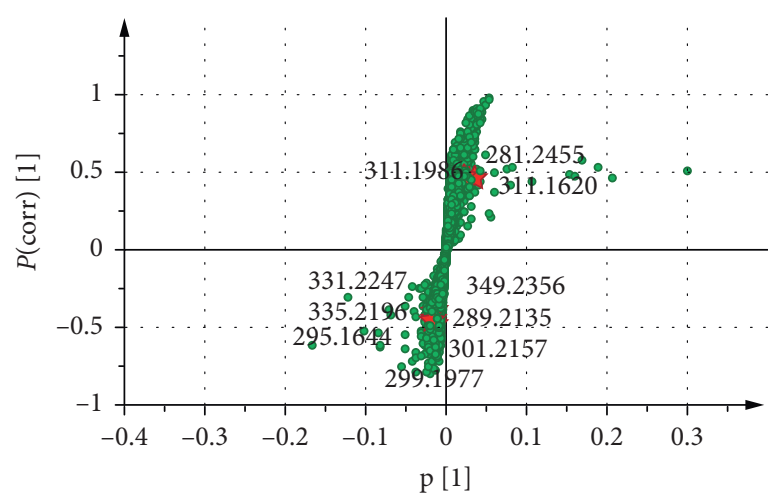

(e)

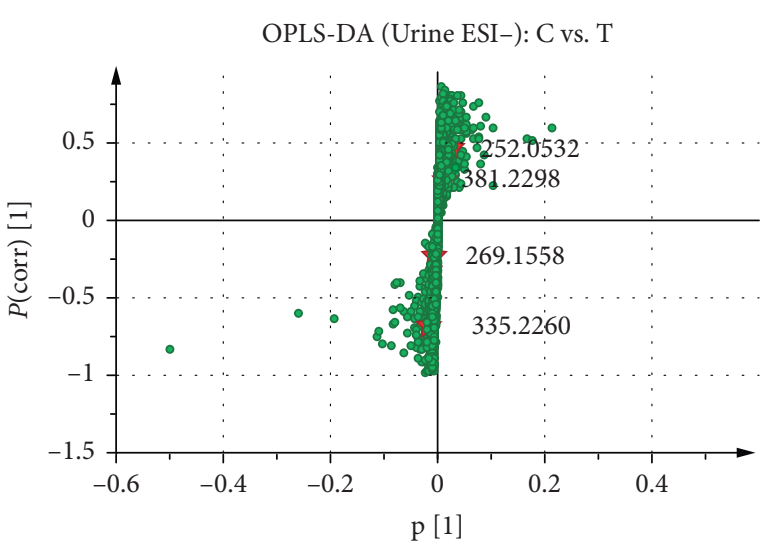

(b)

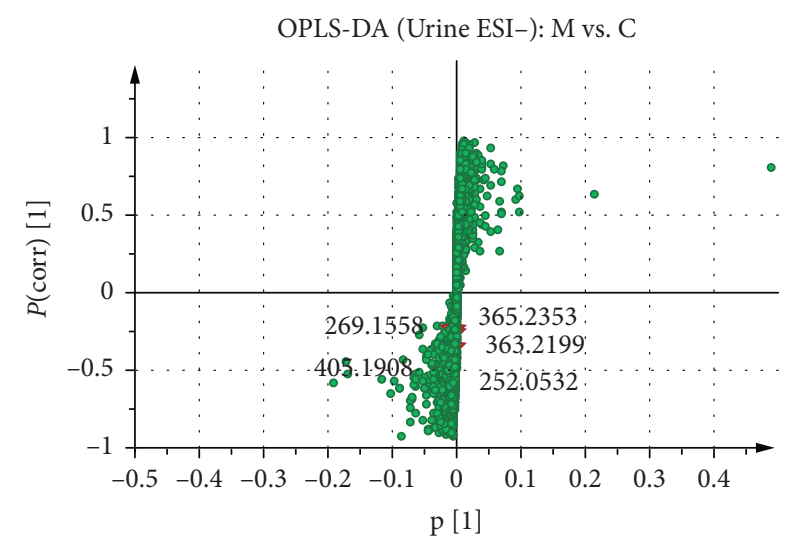

(d)

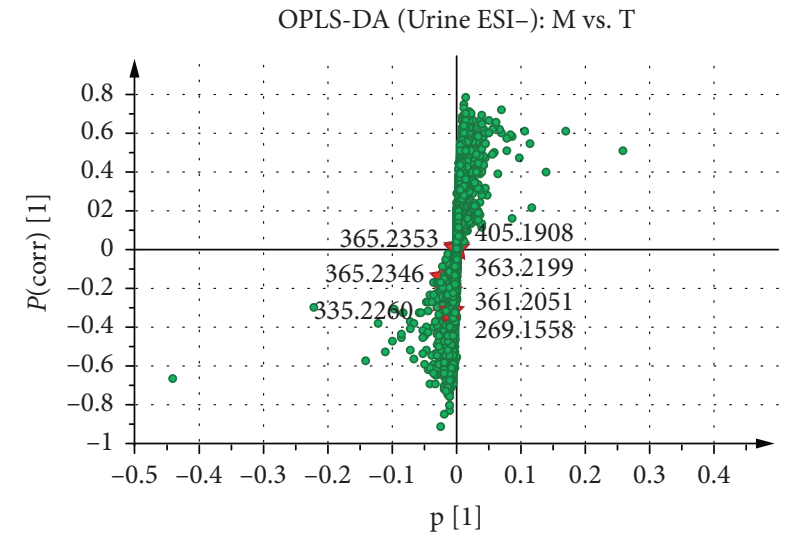

(f)

FIGURE 5: OPLS-DA S-plots of metabolomics analysis based on urine samples. All differential metabolites are shown in S-plots, including the identified results between the control group and the RWCI treatment groups in the ESI ${ }^{+}$mode (a) and in the ESI ${ }^{-}$mode (b), the identified results between the model group and the control group in the $\mathrm{ESI}^{+}$mode (c) and in the $\mathrm{ESI}^{-}$mode (d), and the identified results between the model group and the RWCI treatment groups in the $\mathrm{ESI}^{+}$mode (e) and in the $\mathrm{ESI}^{-}$mode (f). C: control group; M: model group; T: RWCI treatment groups. The $p$ (corr)[1]-axis shows the reliability of each variable in $X$.

Decreased blood flow velocity and increased blood viscosity were main clinical signs of BSS in ancient TCM theory and modern researches [50]. The primary risk factors leading to the occurrence of BSS were anger and sorrow of the seven emotions in TCM theory, and exogenous stimulating factors like cold [51]. Based on this theory, an acute blood stasis rat model was built in the current study.
Increased whole blood viscosity and plasma viscosity showed the success of acute blood stasis rat model establishment. CSMTs have been widely applied in clinical vascular diseases by promoting blood flow and resolving blood stasis in China and other Asian countries [52]; therefore, they were chosen as the positive control drug in the present study. 
TABLE 4: Relative levels of differential metabolites identified in plasma and urine samples.

\begin{tabular}{|c|c|c|c|c|c|}
\hline No. & Levels & $P$ values & No. & Levels & $P$ values \\
\hline P1 & $\mathrm{C}>\mathrm{T}>\mathrm{P}>\mathrm{M}$ & $<0.0001$ & U11 & $\mathrm{M}>\mathrm{T} \approx \mathrm{P} \approx \mathrm{C}$ & 0.0390 \\
\hline $\mathrm{P} 2$ & $\mathrm{C}>\mathrm{T} \approx \mathrm{P}>\mathrm{M}$ & 0.0407 & U12 & $\mathrm{M}>\mathrm{T} \approx \mathrm{P}>\mathrm{C}$ & 0.0045 \\
\hline P3 & $\mathrm{C}>\mathrm{T} \approx \mathrm{P}>\mathrm{M}$ & 0.0093 & U13 & $\mathrm{M}>\mathrm{T} \approx \mathrm{P}>\mathrm{C}$ & 0.0010 \\
\hline $\mathrm{P} 4$ & $\mathrm{C}>\mathrm{T}>\mathrm{P}>\mathrm{M}$ & 0.0207 & U14 & $\mathrm{M}>\mathrm{T}>\mathrm{P}>\mathrm{C}$ & 0.0300 \\
\hline P5 & $\mathrm{M}>\mathrm{T}>\mathrm{P}>\mathrm{C}$ & 0.0012 & U15 & $\mathrm{C}>\mathrm{P} \approx \mathrm{T}>\mathrm{M}$ & 0.0030 \\
\hline P6 & $\mathrm{M}>\mathrm{P}>\mathrm{T}>\mathrm{C}$ & $<0.0001$ & U16 & $\mathrm{C} \approx \mathrm{P} \approx \mathrm{T}>\mathrm{M}$ & 0.0030 \\
\hline P7 & $\mathrm{M}>\mathrm{T} \approx \mathrm{P}>\mathrm{C}$ & 0.0010 & U17 & $\mathrm{M}>\mathrm{T} \approx \mathrm{P} \approx \mathrm{C}$ & 0.0107 \\
\hline P8 & $\mathrm{M}>\mathrm{T}>\mathrm{P}>\mathrm{C}$ & 0.0002 & U18 & $\mathrm{M}>\mathrm{T} \approx \mathrm{P}>\mathrm{C}$ & 0.0156 \\
\hline P9 & $\mathrm{M}>\mathrm{T} \approx \mathrm{P} \approx \mathrm{C}$ & 0.0002 & U19 & $\mathrm{M}>\mathrm{T} \approx \mathrm{P} \approx \mathrm{C}$ & 0.0041 \\
\hline P10 & $\mathrm{M}>\mathrm{T} \approx \mathrm{P}>\mathrm{C}$ & 0.0122 & $\mathrm{U} 20$ & $\mathrm{M}>\mathrm{P}>\mathrm{T}>\mathrm{C}$ & 0.0032 \\
\hline P11 & $\mathrm{C}>\mathrm{T} \approx \mathrm{P}>\mathrm{M}$ & $<0.0001$ & $\mathrm{U} 21$ & $\mathrm{M}>\mathrm{T} \approx \mathrm{P}>\mathrm{C}$ & 0.0385 \\
\hline $\mathrm{P} 12$ & $\mathrm{C} \approx \mathrm{T} \approx \mathrm{P}>\mathrm{M}$ & 0.0006 & $\mathrm{U} 22$ & $\mathrm{M}>\mathrm{T} \approx \mathrm{P} \approx \mathrm{C}$ & 0.0097 \\
\hline P13 & $\mathrm{C} \approx \mathrm{T} \approx \mathrm{P}>\mathrm{M}$ & $<0.0001$ & $\mathrm{U} 23$ & $\mathrm{M}>\mathrm{T} \approx \mathrm{P}>\mathrm{C}$ & 0.0095 \\
\hline P14 & $\mathrm{M}>\mathrm{P}>\mathrm{T}>\mathrm{C}$ & 0.0193 & $\mathrm{U} 24$ & $\mathrm{M}>\mathrm{T}>\mathrm{P}>\mathrm{C}$ & 0.0079 \\
\hline U1 & $\mathrm{M}>\mathrm{P}>\mathrm{T}>\mathrm{C}$ & 0.0047 & $\mathrm{U} 25$ & $\mathrm{M}>\mathrm{P} \approx \mathrm{T}>\mathrm{C}$ & 0.0075 \\
\hline $\mathrm{U} 2$ & $\mathrm{M}>\mathrm{T}>\mathrm{P}>\mathrm{C}$ & $<0.0001$ & $\mathrm{U} 26$ & $\mathrm{M}>\mathrm{T}>\mathrm{P}>\mathrm{C}$ & 0.0137 \\
\hline U3 & $\mathrm{M}>\mathrm{T} \approx \mathrm{P}>\mathrm{C}$ & 0.0141 & $\mathrm{U} 27$ & $\mathrm{M}>\mathrm{P} \approx \mathrm{T}>\mathrm{C}$ & 0.0044 \\
\hline $\mathrm{U} 4$ & $\mathrm{C}>\mathrm{T} \approx \mathrm{P}>\mathrm{M}$ & 0.0001 & $\mathrm{U} 28$ & $\mathrm{M}>\mathrm{P} \approx \mathrm{T}>\mathrm{C}$ & 0.0174 \\
\hline U5 & $\mathrm{M}>\mathrm{T} \approx \mathrm{P}>\mathrm{C}$ & 0.0139 & U29 & $\mathrm{M}>\mathrm{T}>\mathrm{P}>\mathrm{C}$ & 0.0054 \\
\hline U6 & $\mathrm{M}>\mathrm{T} \approx \mathrm{P} \approx \mathrm{C}$ & 0.0076 & U30 & $\mathrm{M}>\mathrm{P}>\mathrm{T}>\mathrm{C}$ & 0.0244 \\
\hline U7 & $\mathrm{M}>\mathrm{T} \approx \mathrm{P} \approx \mathrm{C}$ & 0.0322 & U31 & $\mathrm{M}>\mathrm{P}>\mathrm{T}>\mathrm{C}$ & 0.0092 \\
\hline U8 & $\mathrm{C}>\mathrm{P}>\mathrm{T}>\mathrm{M}$ & 0.0409 & U32 & $\mathrm{M}>\mathrm{P} \approx \mathrm{T}>\mathrm{C}$ & 0.0285 \\
\hline U9 & $\mathrm{C}>\mathrm{P} \approx \mathrm{T}>\mathrm{M}$ & 0.0032 & U33 & $\mathrm{C}>\mathrm{T}>\mathrm{P}>\mathrm{M}$ & 0.0007 \\
\hline U10 & $\mathrm{C}>\mathrm{T} \approx \mathrm{P}>\mathrm{M}$ & 0.0045 & & & \\
\hline
\end{tabular}

The change fold was calculated by getting the average metabolite intensity in corresponding groups of plasma and urine samples and then calculating the ratios of the natural exponential values between the control group and other groups. Multiple-group comparison was performed by ANOVA, thereby getting $P$ values. M: model group; T: RWC treatment groups; P: compound danshen tablet (CDST) group; C: control group.

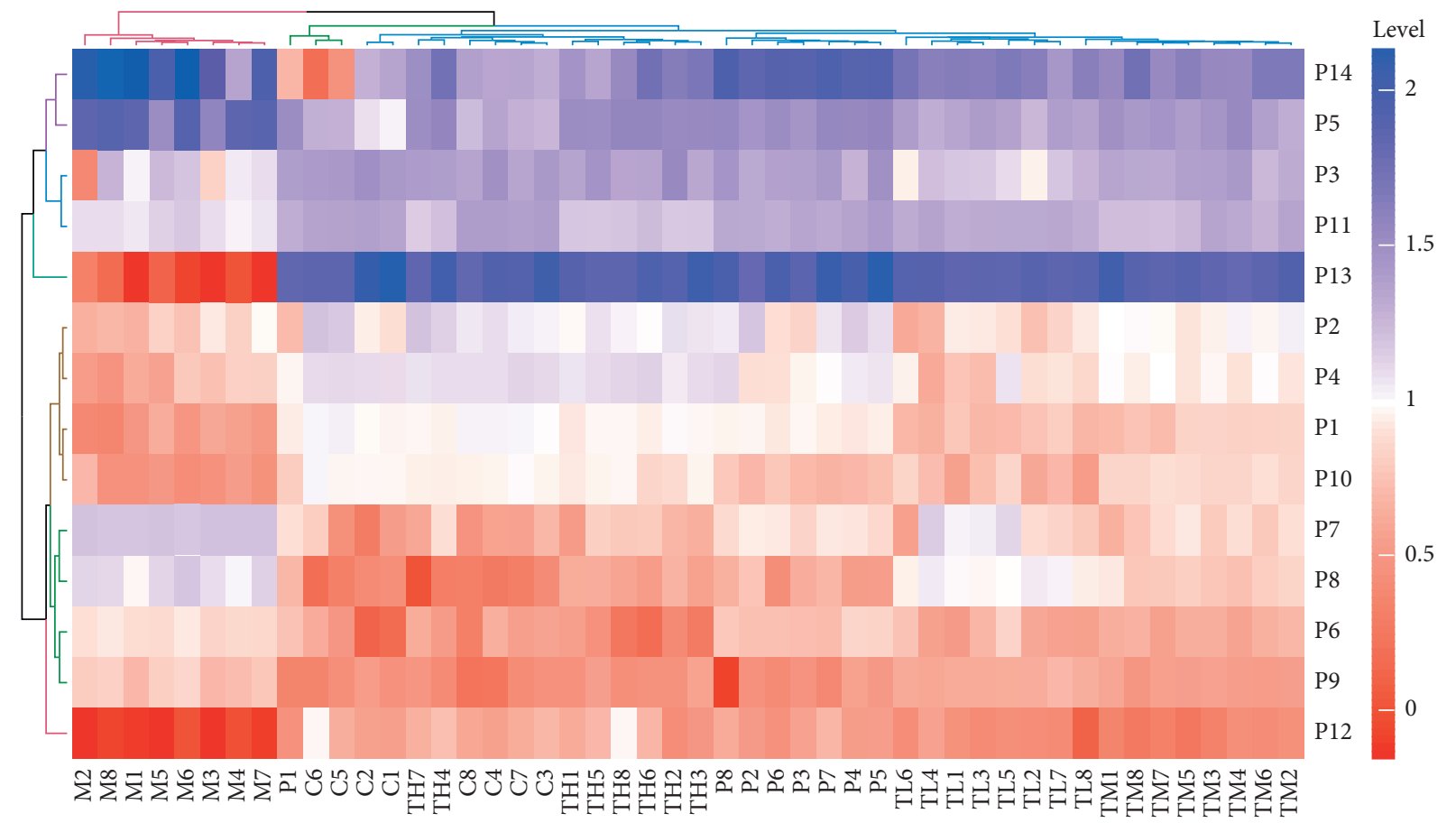

FIGURE 6: Heatmap of differential metabolites identified from plasma samples.

Lipid metabolism, such as steroid hormone biosynthesis, linoleic acid metabolism, alpha-linolenic acid metabolism, arachidonic acid metabolism, biosynthesis of unsaturated fatty acids, and glycerophospholipid metabolism, was perturbed in BSS rats in this study, which was consistent with previous researches $[5,53,54]$. Phosphatidylcholines (PCs) were main phospholipids in the cell membrane structure, involving various biological processes like signal 


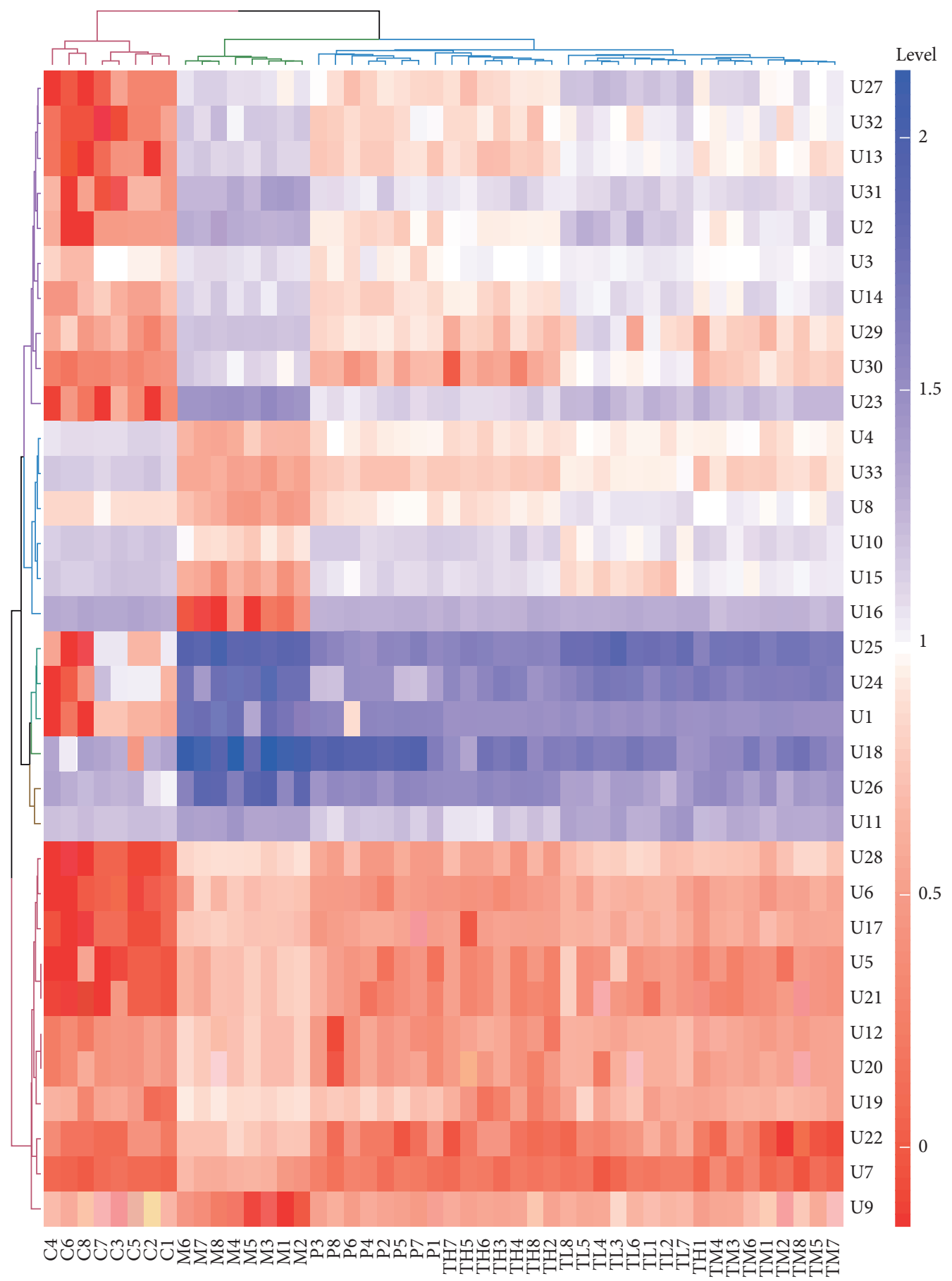

Figure 7: Heatmap of differential metabolites identified from urine samples.

transduction [55] and anti-inflammatory effect [56]. 5 homologues of PCs were identified in plasma samples in the present study, displaying different changing trends. Results showed increased levels of PC (15:0/18:2) and PC (20:4/18:0) as well as the reduced concentrations of PC (14:0/20:3), PC (14:0/20:1), and PC (16:0/20:4) in BSS rats, indicating the metabolic disorder of PCs might be associated with the occurrence and progression of BSS. PCs could be resolved into lysophosphatidylcholine (LysoPC) and unsaturated fatty acids including linoleic acid, alpha-linolenic acid, and arachidonic acid by phospholipase A2 [5]. LysoPC damaged the normal function of vascular endothelial cells and induced thrombogenesis by decreasing the mRNA levels of nitric oxide synthetase and inhibiting the transcriptional activity of nuclear factor kappa-light-chain-enhancer of activated $\mathrm{B}$ cells (NF-kB) and tissue factors.

The pathogenesis of blood stasis was thought to be related to inflammatory factors and immune response in recent studies [57, 58]. Arachidonic acid and its metabolites (eicosanoids) could promote the production of proinflammatory 


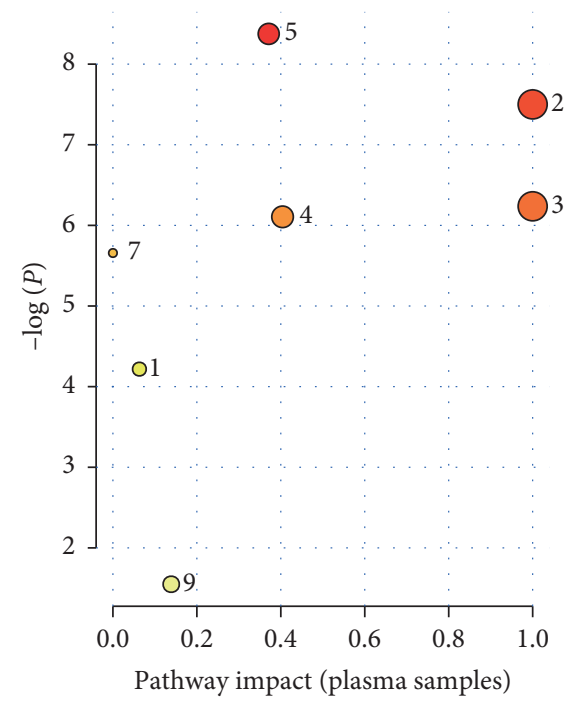

(a)

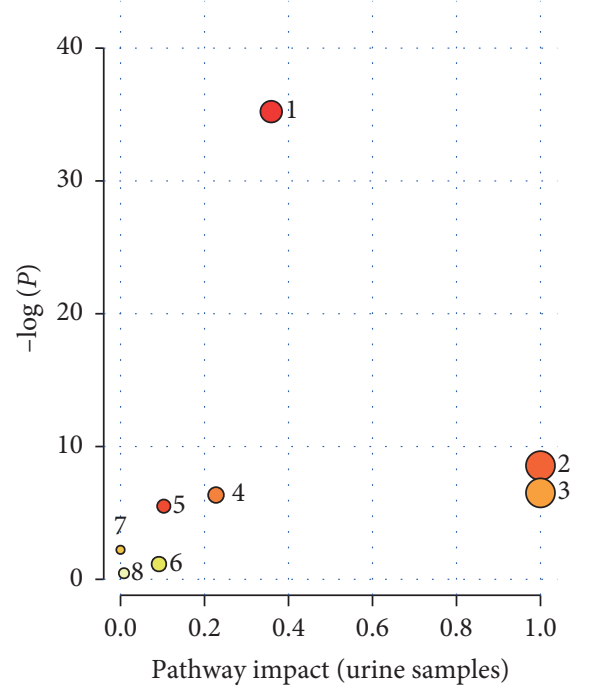

(b)

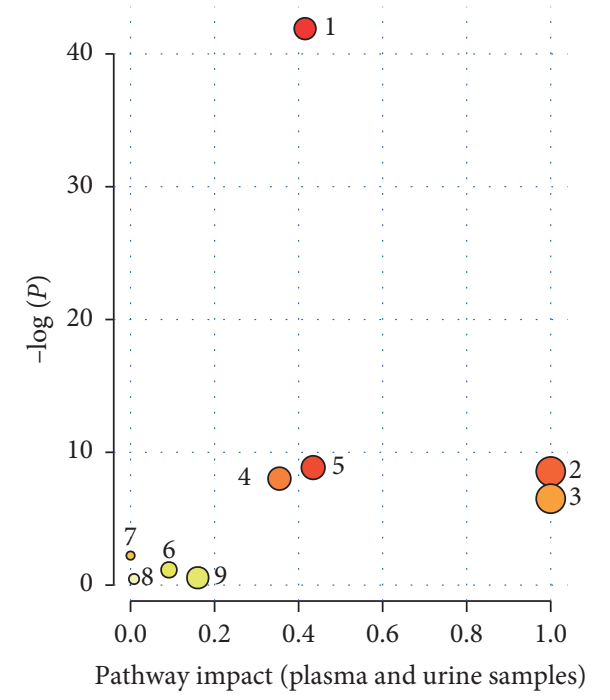

(c)

Figure 8: Metabolic pathway analysis performed by MetaboAnalyst 4.0. Perturbed metabolic pathways were identified in plasma samples (a), urine samples (b), and both plasma and urine samples (c). The numbers represent different metabolic pathways in (a), (b), and (c). 1: steroid hormone biosynthesis; 2: linoleic acid metabolism; 3: alpha-linolenic acid metabolism; 4: retinol metabolism; 5: arachidonic acid metabolism; 6: tryptophan metabolism; 7: biosynthesis of unsaturated fatty acids; 8: steroid biosynthesis; 9: glycerophospholipid metabolism. The values of pathway impact represent the significance of metabolic pathways.

cytokines and inflammatory response [59]. The activation of phospholipase A2 by the protein kinase $\mathrm{C}$ pathway would cause the release of arachidonic acid in the blood stasis state, thereby being resolved into various proinflammatory eicosanoids, like leukotrienes (LTs), prostaglandins (PGs), and thromboxanes by lipoxygenase and cyclooxygenase [60], which might then promote the secretion of inflammatory factors like tumor necrosis factor- $\alpha$ (TNF- $\alpha$ ) and interleukin-1 (IL-1) [37]. Leukotriene a4 (LTA4) hydrolase can catalyze LTA4 into LTB4, an important proinflammatory lipid mediator, which was involved in a series of pathological and physiological processes like the chemotaxis, aggregation, and activation of polymorphonuclear leucocytes and monocytes [61]. In the current study, the levels of arachidonic acid, LTA4,
12(s)-LTB4, 15(s)-HETE, 14,15-epoxy-5,8,11-eicosatrienoic acid, 12(s)-HPETE, and 5-HPETE were found to be higher in BSS model rats than in healthy rats. However, RWCI could improve the fluctuant levels of arachidonic acid and eicosanoids in BSS rats. Therefore, we speculated that the initiation of BSS might be associated with inflammation caused by the disorder of arachidonic acid metabolism. Moreover, RWCI could help prevent these disorders and inhibit the inflammatory state of BSS.

Linoleic acid, a shortest-chain polyunsaturated fatty acid, was the precursor of arachidonic acid. As an essential amino acid, alpha-linolenic acid was employed to produce docosahexaenoic acid and eicosapentaenoic acid (EPA), the latter of which could suppress inflammation by targeting IL- 


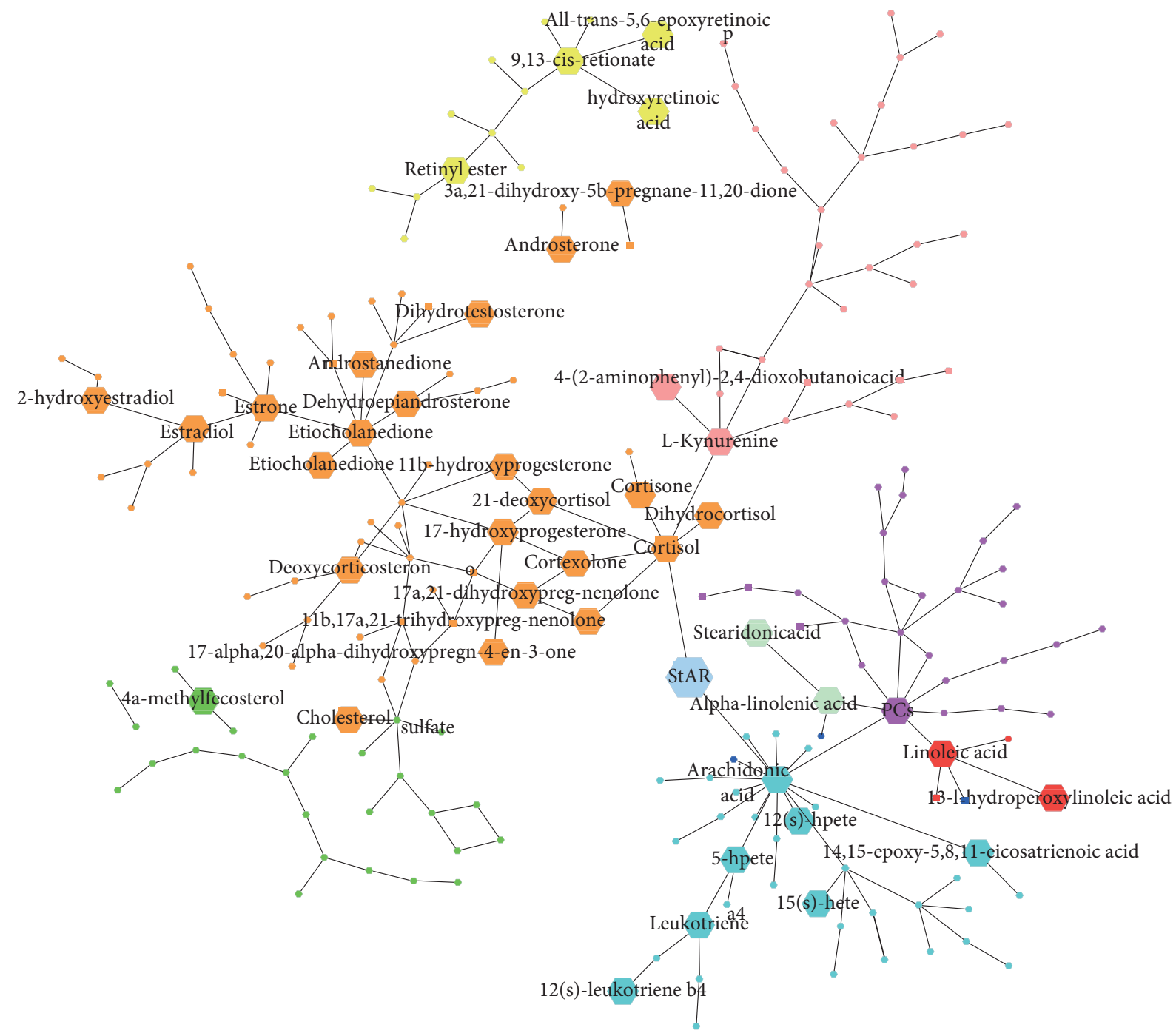

Pathway legends

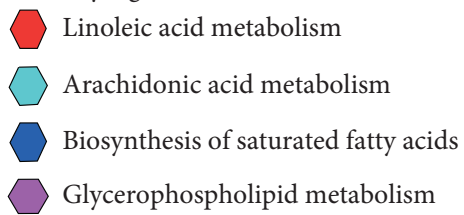

Retinol metabolism

Steroid biosynthesis

Steroid hormone biosynthesis
Tryptophan metabolism

Alpha-linolenic acid metabolism

StAR

FIGURE 9: Metabolic network of all differential metabolites identified in plasma and urine samples. StAR: steroidogenic acute regulatory protein.

6 and TNF- $\alpha$ [62]. Stearidonic acid was a metabolic intermediate in the conversion process of alpha-linolenic acid and EPA. Concentrations of linoleic acid, alpha-linolenic acid, and stearidonic acid were decreased in rats with BSS compared with healthy rats. However, the disturbance of these levels could be ameliorated after RWCI treatment. In previous research, metabolic disorders of linoleic acid and alpha-linolenic acid were also found in BSS and were then improved by TCM, for example, the treatment of the acute blood stasis model rat with Taohong Siwu decoction [63].

Steroid hormone biosynthesis mainly included the production of androgens and estrogens. Steroidogenic acute regulatory protein (StAR) could mediate cholesterol into mitochondria to synthesize pregnenolone [64], which was regulated by arachidonic acid- and cyclic adenosine monophosphate-transduced signals [65]. Cortisol had an important effect on regulating the stress response of the organism [66]. A study indicated that hydrocortisone could amplify the responses of the leg vasoconstrictor to the cold pressor test and vascular smooth muscle to catecholamines [67]. Dehydroepiandrosterone sulfate, converted from dehydroepiandrosterone, could inhibit inflammation in atherosclerosis by activating peroxisome proliferator-activated receptors and regulating the $N F-\kappa B$ signal pathway [68]. In addition, the disorder of steroid hormone biosynthesis was related to primary dysmenorrhea accompanied by BSS [69]. Steroid 
hormones levels were increased in acute blood stasis model rats compared with healthy rats and were improved with RWCI treatment, indicating that inflammation and endocrine disorder might participate in the development of BSS. These results were in accord with those of previous researches $[54,70]$.

Retinoic acids were involved in various cell activities in the biological process, for example, the secretion of matrix metalloproteinase, cell communication and differentiation, and the expression of inflammatory factors like IL- 6 and TNF- $\alpha$ [71]. Previous studies have shown that the decreased levels of retinoic acid in asthma subjects might lead to airway hyperresponsiveness and accelerate disease progression by activating NF- $\mathrm{NB}[72,73]$. In the present study, the levels of compounds participated in retinol metabolism, such as alltrans-retinoic acid, 9-cis-retinoic acid, retinyl ester, and hydroxyretinoic acid, were decreased in BSS rats. Moreover, their levels were increased after RWCI therapy. These results indicated RWCI might improve retinol metabolism disorder, thereby relieving the syndrome of blood stasis.

Kynurenine, as a main catabolite of tryptophan, participated in the regulation of innate and adaptive immunity in biological processes. It might inhibit the proliferation of immune cells such as $\mathrm{T}$ cells and natural killer cells. In addition, it could negatively modulate immunogenic dendritic cells [74]. Tryptophan metabolism had a potential correlation with blood stasis in the current research. The increased level of kynurenine in BSS rats could be improved by RWCI treatment.

\section{Conclusions}

In the current study, metabolomics was utilized to explore the pathogenesis of BSS and pharmacological effect of RWCI. The change of clinical manifestations and signs, hemorheological parameter, and inflammation in BSS rats could be markedly improved after RWCI treatment. 14 and 33 differential metabolites in plasma and urine samples were identified by metabolomics based on UPLC-Q/TOF-MS, mainly involving 9 disturbed metabolic pathways. Thereinto, 5 metabolic pathways (steroid hormone biosynthesis, linoleic acid metabolism, alpha-linolenic acid metabolism, retinol metabolism, and arachidonic acid metabolism) were significantly related to the occurrence of BSS. The pathogenesis of BSS might be associated with inflammatory responses and endocrine disorder. However, RWCI had a potential to prevent the disorder of metabolic pathways, indicating RWCI might be considered a novel TCM to ameliorate BSS. These results might provide corresponding experimental basis for clinical application. Subsequent researches should further explore the changes of genes and proteins associated with disturbed metabolic pathways, thereby uncovering relevant signal pathways related to the occurrence of BSS.

\section{Data Availability}

The original data employed to support the results of this study can be obtained from the corresponding author upon request.

\section{Conflicts of Interest}

The authors declare that there are no conflicts of interest regarding the publication of this study.

\section{Acknowledgments}

This research was funded by the Key Laboratory of Health and Family Planning Commission of Jilin Province of China (Grant no. 3D5172303426) and Provincial School CoConstruction Industrialization Demonstration Project of Jilin Province (Grant no. SXGJSF2017-1-1(01)). The authors would like to thank Hongqiang Lin from the Research Center of Natural Drug, School of Pharmaceutical Sciences, Jilin University, for providing help in the metabolomics research work.

\section{Supplementary Materials}

Tables S1 and S2 are used to validate the systematic stability and repeatability of UPLC-Q/TOF-MS during the test of plasma and urine samples by calculating repeatability and intermediate precision. Figure S1 shows PCA results of plasma and urine samples in $\mathrm{ESI}^{+}$and $\mathrm{ESI}^{-}$modes based on UPLC-Q/TOF-MS. Table S3 shows the details of quality parameters (R2X, R2Y, and Q2) from cross-validation of the OPLS-DA model. The pairwise-comparison $p$ values of the post hoc analysis for multiple comparisons in rats are shown in Table S4. Table S5 indicates the relative information of perturbed metabolic pathways in plasma and urine samples. Figures S2 to S16 show mass spectra of all 14 metabolites identified in plasma samples. Among them, retinoic acids include all-trans-retinoic acid (Figure S4) and 9-cis-retinoic acid (Figure S5). Figures S17 to S51 show mass spectra of all 33 metabolites identified in plasma samples. Among them, retinoic acids include all-trans-retinoic acid (Figure S26) and 9-cis-retinoic acid (Figure S27). HPETEs include 12(s)HPETE (Figure S48) and 5-HPETE (Figure S49). The ROC curve is used to evaluate the accuracy of distinct metabolites identified in plasma and urine samples as potential biomarkers (see Figures S52 and S53). (Supplementary Materials)

\section{References}

[1] A. J. Alves, J. L. Viana, S. L. Cavalcante et al., "Physical activity in primary and secondary prevention of cardiovascular disease: overview updated," World Journal of Cardiology, vol. 8, no. 10, pp. 575-583, 2016.

[2] W. Lei, J. Ni, X. Xia, M. Jiang, and G. Bai, "Searching for synergistic calcium antagonists and novel therapeutic regimens for coronary heart disease therapy from a traditional Chinese medicine, Suxiao Jiuxin Pill," Journal of Chromatography B, vol. 1092, pp. 220-227, 2018.

[3] T. H. Lan, L. L. Zhang, Y. H. Wang, H. L. Wu, and D. P. Xu, "Systems pharmacology dissection of traditional Chinese medicine Wen-Dan decoction for treatment of cardiovascular diseases," Evidence-Based Complementary and Alternative Medicine, vol. 2018, Article ID 5170854, 14 pages, 2018. 
[4] X. Dang, J. J. Miao, A. Q. Chen et al., "The antithrombotic effect of RSNK in blood-stasis model rats," Journal of Ethnopharmacology, vol. 173, pp. 266-272, 2015.

[5] Z. Yuan, L. Zhong, Y. Hua et al., "Metabolomics study on promoting blood circulation and ameliorating blood stasis: investigating the mechanism of Angelica sinensis and its processed products," Biomedical Chromatography, vol. 33, no. 4, p. e4457, 2019.

[6] S. Su, J. Duan, W. Cui et al., "Network-based biomarkers for cold coagulation blood stasis syndrome and the therapeutic effects of Shaofu Zhuyu decoction in rats," Evidence-Based Complementary and Alternative Medicine, vol. 2013, Article ID 901943, 15 pages, 2013.

[7] M. H. Cha, M. J. Kim, J. Jung, J. H. Kim, M. S. Lee, and M. S. Kim, "Metabolomic analysis of clinical plasma from cerebral infarction patients presenting with blood stasis," Evidence-Based Complementary and Alternative Medicine, vol. 2015, Article ID 453423, 7 pages, 2015.

[8] L. He, M. Fang, L. Chen et al., "Transcriptome analysis of blood stasis syndrome in subjects with hypertension," Journal of Traditional Chinese Medicine, vol. 36, no. 2, pp. 173-180, 2016.

[9] K. J. Chen, "Blood stasis syndrome and its treatment with activating blood circulation to remove blood stasis therapy," Chinese Journal of Integrative Medicine, vol. 18, no. 12, pp. 891-896, 2012.

[10] Y. Wang, J. Yan, S. Li et al., "Composition of the essential oil from Danggui-Zhiqiao herb-pair and its analgesic activity and effect on hemorheology in rats with blood stasis syndrome," Pharmacognosy Magazine, vol. 12, no. 48, pp. 271-275, 2016.

[11] G. G. Yousef, M. H. Grace, D. M. Cheng, I. V. Belolipov, I. Raskin, and M. A. Lila, "Comparative phytochemical characterization of three Rhodiola species," Phytochemistry, vol. 67, no. 21, pp. 2380-2391, 2006.

[12] H. M. Chiang, H. C. Chen, C. S. Wu, P. Y. Wu, and K. C. Wen, "Rhodiola plants: chemistry and biological activity," Journal of Food and Drug Analysis, vol. 23, no. 3, pp. 359-369, 2015.

[13] M. Grech-Baran, K. Syklowska-Baranek, and A. Pietrosiuk, "Approaches of Rhodiola kirilowii and Rhodiola rosea field cultivation in Poland and their potential health benefits," Annals of Agricultural and Environmental Medicine, vol. 22, no. 2, pp. 281-285, 2015.

[14] Y. Liu, C. Chen, J. Qiu et al., "Characterization of the chemical constituents in Hongjingtian injection by liquid chromatography quadrupole time-of-flight mass spectrometry," Biomedical Chromatography, vol. 33, no. 3, p. e4446, 2019.

[15] L. Yao, L. Nie, Y. Zhang, Y. Liu, and M. Cao, "Study on fingerprint of Dazhu Hongjingtian injection," Chinese Pharmaceutical Affairs, vol. 25, no. 7, pp. 916-918, 2011.

[16] R. Wojcik, A. K. Siwicki, E. Skopinska-Rozewska, A. Wasiutynski, E. Sommer, and M. Furmanowa, "The effect of Chinese medicinal herb Rhodiola kirilowii extracts on cellular immunity in mice and rats," Polish Journal of Veterinary Sciences, vol. 12, no. 3, pp. 399-405, 2009.

[17] L. Chen, B. Yu, Y. Zhang et al., "Bioactivity-guided fractionation of an antidiarrheal Chinese herb Rhodiola kirilowii (Regel) Maxim reveals (-)-epicatechin-3-gallate and (-)-epigallocatechin-3-gallate as inhibitors of cystic fibrosis transmembrane conductance regulator," PLoS One, vol. 10, no. 3, Article ID e0119122, 2015.

[18] L. Yu, Y. Qin, Q. Wang et al., "The efficacy and safety of Chinese herbal medicine, Rhodiola formulation in treating ischemic heart disease: a systematic review and meta-analysis of randomized controlled trials," Complementary Therapies in Medicine, vol. 22, no. 4, pp. 814-825, 2014.

[19] G. M. Shen and X. S. Tian, "Influence of big plant Rhodiola capsule treat on unstable angina patients," Chinese Journal of Experimental Traditional Medical Formulae, vol. 20, no. 16, pp. 200-203, 2014.

[20] T. F. Jorge, J. A. Rodrigues, C. Caldana et al., "Mass spectrometry-based plant metabolomics: metabolite responses to abiotic stress," Mass Spectrometry Reviews, vol. 35, no. 5, pp. 620-649, 2016.

[21] L. Qin, Z. Zhang, M. Guo et al., "Plasma metabolomics combined with lipidomics profiling reveals the potential antipyretic mechanisms of Qingkailing injection in a rat model," Chemico-Biological Interactions, vol. 254, pp. 24-33, 2016.

[22] J. Krumsiek, J. Bartel, and F. J. Theis, "Computational approaches for systems metabolomics," Current Opinion in Biotechnology, vol. 39, pp. 198-206, 2016.

[23] M. Wang, L. Chen, D. Liu, H. Chen, D. D. Tang, and Y. Y. Zhao, "Metabolomics highlights pharmacological bioactivity and biochemical mechanism of traditional Chinese medicine," Chemico-Biological Interactions, vol. 273, pp. 133-141, 2017.

[24] Y. Y. Zhao, P. Lei, D. Q. Chen, Y. L. Feng, and X. Bai, "Renal metabolic profiling of early renal injury and renoprotective effects of Poria cocos epidermis using UPLC Q-TOF/HSMS/ MSE," Journal of Pharmaceutical and Biomedical Analysis, vol. 81-82, pp. 202-209, 2013.

[25] Y. Y. Zhao and R. C. Lin, "UPLC-MS(E) application in disease biomarker discovery: the discoveries in proteomics to metabolomics," Chemico-Biological Interactions, vol. 215, pp. 7-16, 2014.

[26] Y. Y. Zhao, X. L. Cheng, F. Wei et al., "Intrarenal metabolomic investigation of chronic kidney disease and its TGF- $\beta 1$ mechanism in induced-adenine rats using UPLC Q-TOF/ HSMS/MS ${ }^{\mathrm{E}}$," Journal of Proteome Research, vol. 12, no. 2, pp. 692-703, 2013.

[27] N. P. Committee, Pharmacopoeia of the People's Republic of China, Volume I, China Medico-Pharmaceutical Science \& Technology Publishing House, Beijing, China, 1954.

[28] W. Wei, W. Ximei, and L. Yuanjian, Experimental Methodology of Pharmacology, People's Medical Publishing House, Beijing, China, 2010.

[29] X. Zhang, C. Zhang, J. Sai et al., "Xueshuan Xinmaining tablet treats blood stasis through regulating the expression of F13a1, Car1, and Tbxa2r," Evidence-Based Complementary and Alternative Medicine, vol. 2015, Article ID 704390, 8 pages, 2015.

[30] L. Xiong, J. Xie, C. Song et al., "The activation of Nrf2 and its downstream regulated genes mediates the antioxidative activities of Xueshuan Xinmaining tablet in human umbilical vein endothelial cells," Evidence-Based Complementary and Alternative Medicine, vol. 2015, Article ID 187265, 7 pages, 2015.

[31] Z. Pang, G. Wang, C. Wang, W. Zhang, J. Liu, and F. Wang, "Serum metabolomics analysis of asthma in different inflammatory phenotypes: a cross-sectional study in northeast China," BioMed Research International, vol. 2018, Article ID 2860521, 14 pages, 2018.

[32] D. S. Wishart, Y. D. Feunang, A. Marcu et al., "HMDB 4.0: the human metabolome database for 2018," Nucleic Acids Research, vol. 46, no. D1, pp. D608-D617, 2018.

[33] J. Chong and J. Xia, "MetaboAnalystR: an R package for flexible and reproducible analysis of metabolomics data," Bioinformatics, vol. 34, no. 24, pp. 4313-4314, 2018. 
[34] M. Kanehisa, Y. Sato, M. Furumichi, K. Morishima, and M. Tanabe, "New approach for understanding genome variations in KEGG," Nucleic Acids Research, vol. 47, no. D1, pp. D590-D595, 2019.

[35] V. Samanidou, K. Pantazidou, L. Kovatsi, S. Njau, and A. Livanos, "A simple HPLC method for the simultaneous determination of two selective serotonin reuptake inhibitors and two serotonin-norepinephrine reuptake inhibitors in hair, nail clippings, and cerebrospinal fluid," Journal of Separation Science, vol. 35, no. 7, pp. 839-845, 2012.

[36] H. Wu, Y. Chen, Z. Li, and X. Liu, "Untargeted metabolomics profiles delineate metabolic alterations in mouse plasma during lung carcinoma development using UPLC-QTOF/MS in MS(E) mode," Royal Society Open Science, vol. 5, no. 9, Article ID 181143, 2018.

[37] M. Hao, D. Ji, L. Li et al., "Mechanism of curcuma wenyujin rhizoma on acute blood stasis in rats based on a UPLC-Q/ TOF-MS metabolomics and network approach," Molecules, vol. 24, no. 1, p. 82, 2018.

[38] N. Ma, I. Karam, X. W. Liu et al., "UPLC-Q-TOF/MS-based urine and plasma metabonomics study on the ameliorative effects of aspirin eugenol ester in hyperlipidemia rats," Toxicology and Applied Pharmacology, vol. 332, pp. 40-51, 2017.

[39] A. H. Zhang, Z. M. Ma, H. Sun et al., "High-throughput metabolomics evaluate the efficacy of total lignans from Acanthophanax senticosus stem against ovariectomized osteoporosis rat," Frontiers in Pharmacology, vol. 10, p. 553, 2019.

[40] A. R. H. Farrag, H. M. I. Abdallah, A. R. Khattab et al., "Antiulcer activity of Cyperus alternifolius in relation to its UPLC-MS metabolite fingerprint: a mechanistic study," Phytomedicine, vol. 62, Article ID 152970, , 2019.

[41] Z. Pang, G. Wang, N. Ran et al., "Inhibitory effect of methotrexate on rheumatoid arthritis inflammation and comprehensive metabolomics analysis using ultra-performance liquid chromatography-quadrupole time of flightmass spectrometry (UPLC-Q/TOF-MS)," International Journal of Molecular Sciences, vol. 19, no. 10, p. 2894, 2018.

[42] D. Q. Chen, H. Chen, L. Chen, D. D. Tang, H. Miao, and Y. Y. Zhao, "Metabolomic application in toxicity evaluation and toxicological biomarker identification of natural product," Chemico-Biological Interactions, vol. 252, pp. 114-130, 2016.

[43] Y. Y. Zhao and R. C. Lin, "Metabolomics in nephrotoxicity," in Advances in Clinical Chemistry, vol. 65, pp. 69-89, Elsevier, Amsterdam, Netherlands, 2014.

[44] Y. Y. Zhao, D. D. Tang, H. Chen et al., "Urinary metabolomics and biomarkers of aristolochic acid nephrotoxicity by UPLCQTOF/HDMS," Bioanalysis, vol. 7, no. 6, pp. 685-700, 2015.

[45] Y. Y. Zhao, H. L. Wang, X. L. Cheng et al., "Metabolomics analysis reveals the association between lipid abnormalities and oxidative stress, inflammation, fibrosis and Nrf2 dysfunction in aristolochic acid-induced nephropathy," Scientific Reports, vol. 5, no. 1, p. 12936, 2015.

[46] C. Y. Nie, T. Han, L. Zhang et al., "Cross-sectional and dynamic change of serum metabolite profiling for Hepatitis B-related acute-on-chronic liver failure by UPLC/MS," Journal of Viral Hepatitis, vol. 21, no. 1, pp. 53-63, 2014.

[47] Y. I. Cho and D. J. Cho, "Hemorheology and microvascular disorders," Korean Circulation Journal, vol. 41, no. 6, pp. 287-295, 2011.

[48] Q. Ma, P. L. Li, Y. L. Hua et al., "Effects of Tao-Hong-Si-Wu decoction on acute blood stasis in rats based on a LC-Q/TOF-
MS metabolomics and network approach," Biomedical Chromatography, vol. 32, no. 4, p. e4144, 2018.

[49] M. Hayakawa, K. Maekawa, S. Kushimoto et al., "High $\mathrm{D}$-dimer levels predict a poor outcome in patients with severe trauma, even with high fibrinogen levels on Arribal: a multicenter reterospective study," Shock, vol. 45, no. 3, pp. 308314, 2016.

[50] L. He, H. Wang, C. Gu, X. He, L. Zhao, and X. Tong, “Administration of traditional Chinese blood circulation activating drugs for microvascular complications in patients with type 2 diabetes mellitus," Journal of Diabetes Research, vol. 2016, Article ID 1081657, 9 pages, 2016.

[51] G. Xie, S. Hao, Z. Ma et al., "Effect of Qingnao tablet on blood viscosity of rat model of blood stasis induced by epinephrine," AIP Conference Proceedings, vol. 1956, no. 1, pp. 020037020031, 2018.

[52] K. Wang, D. Zhang, J. Wu, S. Liu, X. Zhang, and B. Zhang, "A comparative study of Danhong injection and Salvia miltiorrhiza injection in the treatment of cerebral infarction: a systematic review and meta-analysis," Medicine, vol. 96, no. 22, p. e7079, 2017.

[53] D. Cao, C. Xu, Y. Xue et al., "The therapeutic effect of Ilex pubescens extract on blood stasis model rats according to serum metabolomics," Journal of Ethnopharmacology, vol. 227, pp. 18-28, 2018.

[54] P. Liu, E.-X. Shang, Y. Zhu, J.-G. Yu, D.-W. Qian, and J. A. Duan, "Comparative analysis of compatibility effects on invigorating blood circulation for Cyperi rhizoma series of herb pairs using untargeted metabolomics," Frontiers in Pharmacology, vol. 8, p. 677, 2017.

[55] A. M. Crane, H. U. Hua, A. D. Coggin, B. G. Gugiu, B. L. Lam, and S. K. Bhattacharya, "Mass spectrometric analyses of phosphatidylcholines in alkali-exposed corneal tissue," Investigative Opthalmology \& Visual Science, vol. 53, no. 11, pp. 7122-7130, 2012.

[56] P. Hartmann, A. Szabó, G. Erős et al., “Anti-inflammatory effects of phosphatidylcholine in neutrophil leukocyte-dependent acute arthritis in rats," European Journal of Pharmacology, vol. 622, no. 1-3, pp. 58-64, 2009.

[57] X.-H. Wu, C. Zhao, A.-H. Zhang et al., "High-throughput metabolomics used to identify potential therapeutic targets of Guizhi Fuling Wan against endometriosis of cold coagulation and blood stasis," RSC Advances, vol. 8, no. 34, pp. 1923819250, 2018.

[58] C. Y. Ma, J. H. Liu, J. X. Liu et al., "Relationship between two blood stasis syndromes and inflammatory factors in patients with acute coronary syndrome," Chinese Journal of Integrative Medicine, vol. 23, no. 11, pp. 845-849, 2017.

[59] P. C. Calder, "Omega-3 fatty acids and inflammatory processes: from molecules to man," Biochemical Society Transactions, vol. 45, no. 5, pp. 1105-1115, 2017.

[60] H. Meng, Y. Liu, and L. Lai, "Diverse ways of perturbing the human arachidonic acid metabolic network to control inflammation," Accounts of Chemical Research, vol. 48, no. 8, pp. 2242-2250, 2015.

[61] M. Bäck, C. Weber, and E. Lutgens, "Regulation of atherosclerotic plaque inflammation," Journal of Internal Medicine, vol. 278, no. 5, pp. 462-482, 2015.

[62] E. Tortosa-Caparrós, D. Navas-Carrillo, F. Marin, and E. Orenes-Piñero, "Anti-inflammatory effects of omega 3 and omega 6 polyunsaturated fatty acids in cardiovascular disease and metabolic syndrome," Critical Reviews in Food Science and Nutrition, vol. 57, no. 16, pp. 3421-3429, 2017. 
[63] X. Zhang, P. Li, Y. Hua et al., "Urinary metabolomics study the mechanism of Taohong Siwu Decoction intervention in acute blood stasis model rats based on liquid chromatography coupled to quadrupole time-of-flight mass spectrometry," Journal of Chromatography B, vol. 1074-1075, pp. 51-60, 2018.

[64] D. M. Stocco, "StAR protein and the regulation of steroid hormone biosynthesis," Annual Review of Physiology, vol. 63, no. 1, pp. 193-213, 2001.

[65] X. Wang, L. P. Walsh, A. J. Reinhart, and D. M. Stocco, "The role of arachidonic acid in steroidogenesis and steroidogenic acute regulatory (StAR) gene and protein expression," Journal of Biological Chemistry, vol. 275, no. 26, pp. 20204-20209, 2000.

[66] J. V. Zorn, R. R. Schür, M. P. Boks, R. S. Kahn, M. Joëls, and C. H. Vinkers, "Cortisol stress reactivity across psychiatric disorders: a systematic review and meta-analysis," Psychoneuroendocrinology, vol. 77, pp. 25-36, 2017.

[67] A. M. Adlan, J. C. S. V. van Zanten, G. Y. H. Lip, J. F. R. Paton, G. D. Kitas, and J. P. Fisher, "Acute hydrocortisone administration reduces cardiovagal baroreflex sensitivity and heart rate variability in young men," The Journal of Physiology, vol. 596, no. 20, pp. 4847-4861, 2018.

[68] X. Cai, J. Du, L. Li et al., "Clinical metabolomics analysis of therapeutic mechanism of Tongmai Yangxin Pill on stable angina," Journal of Chromatography B, vol. 1100-1101, pp. 106-112, 2018.

[69] P. Liu, J. Duan, P. Wang et al., "Biomarkers of primary dysmenorrhea and herbal formula intervention: an exploratory metabonomics study of blood plasma and urine," Molecular BioSystems, vol. 9, no. 1, pp. 77-87, 2013.

[70] X. Huang, S. Su, J. A. Duan et al., "Effects and mechanisms of Shaofu-Zhuyu decoction and its major bioactive component for cold-stagnation and blood-stasis primary dysmenorrhea rats," Journal of Ethnopharmacology, vol. 186, pp. 234243, 2016.

[71] M. A. Kane, N. Sidell, and R. N. Taylor, "Pathogenesis of endometriosis: roles of retinoids and inflammatory pathways," Seminars in Reproductive Medicine, vol. 33, no. 4, pp. 246-256, 2015.

[72] R. Reifen, Z. Berkovich, and A. Mandelberg, "Vitamin A supplementation via aerosol spray in asthmatic children," Pediatric Allergy and Immunology, vol. 26, no. 6, pp. 578-579, 2015.

[73] D. M. Schwartz, T. K. Farley, N. Richoz et al., "Retinoic acid receptor alpha represses a Th9 transcriptional and epigenomic program to reduce allergic pathology," Immunity, vol. 50, no. 1, pp. 106 e110-120 e110, 2019.

[74] G. Liu, S. Chen, J. Zhong, K. Teng, and Y. Yin, "Crosstalk between tryptophan metabolism and cardiovascular disease, mechanisms, and therapeutic implications," Oxidative Medicine and Cellular Longevity, vol. 2017, Article ID 1602074, 5 pages, 2017. 


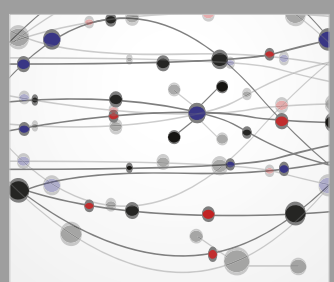

The Scientific World Journal
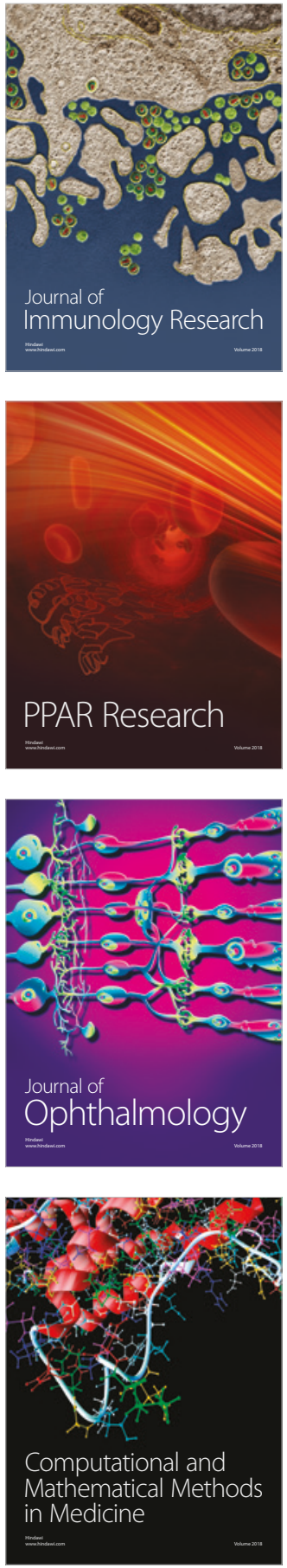

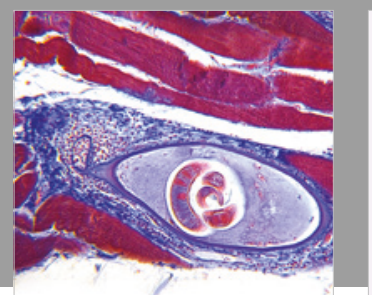

Gastroenterology Research and Practice

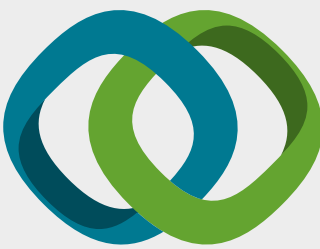

\section{Hindawi}

Submit your manuscripts at

www.hindawi.com
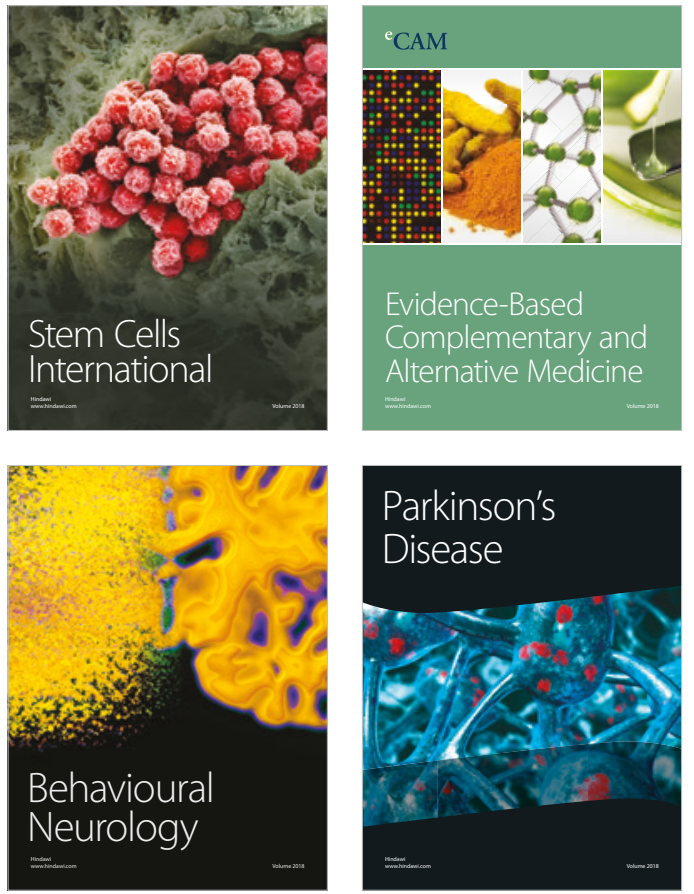

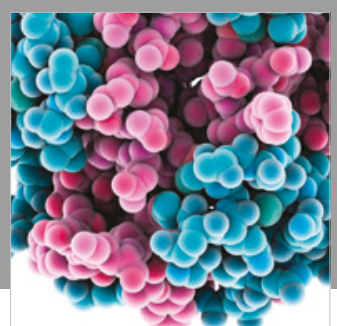

ournal of

Diabetes Research

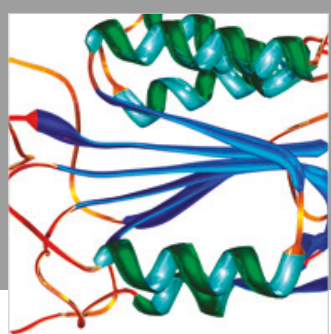

Disease Markers
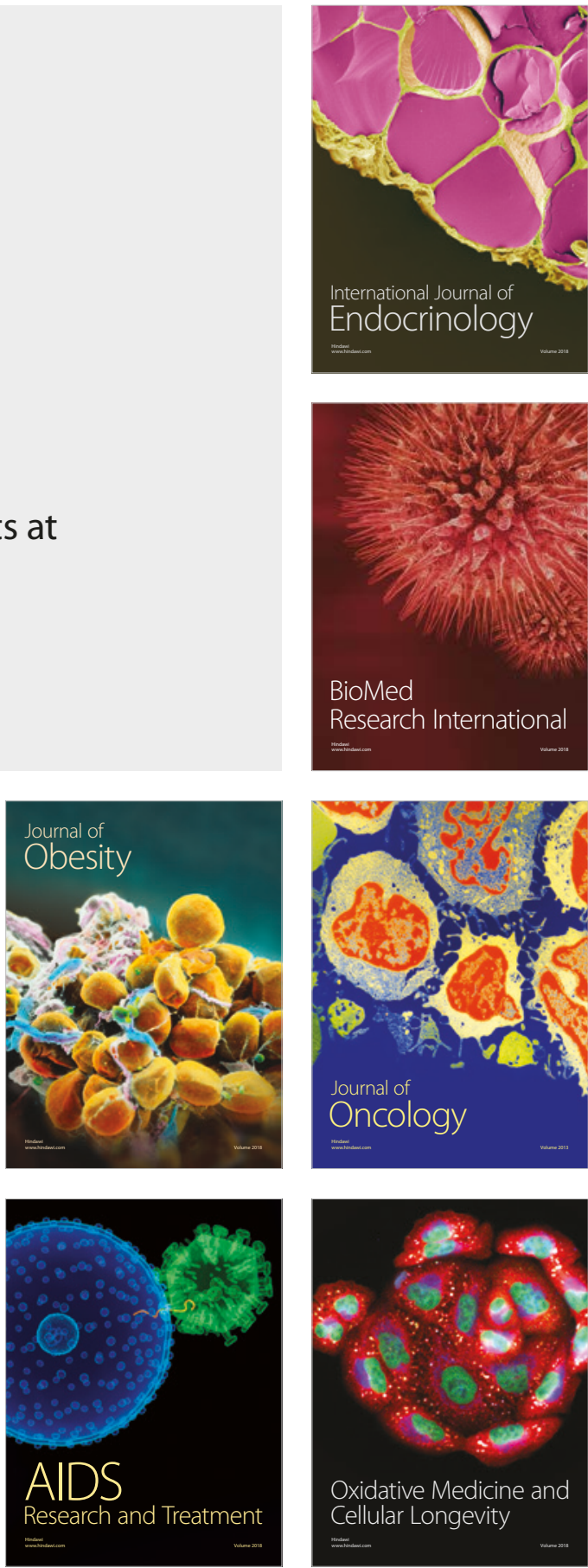